\title{
Diffusion in a Locally Stationary Random Environment
}

\author{
Rémi Rhodes*
}

December 18, 2007

\begin{abstract}
This paper deals with homogenization of diffusion processes in a locally stationary random environment. Roughly speaking, such an environment possesses two evolution scales: both a fast microscopic one and a smoothly varying macroscopic one. The homogenization procedure aims at giving a macroscopic approximation that takes into account the microscopic heterogeneities.
\end{abstract}

\section{Introduction}

In this paper, we aim at describing the asymptotic behavior (homogenization), as the parameter $\varepsilon$ tends to 0 , of the solution $X^{\varepsilon}$ of the following Stochastic Differential Equation (SDE)

(1) $X_{t}^{\varepsilon}=x+\frac{1}{\varepsilon} \int_{0}^{t} b\left(\omega, X_{r}^{\varepsilon} / \varepsilon, X_{r}^{\varepsilon}\right) d r+\int_{0}^{t} c\left(\omega, X_{r}^{\varepsilon} / \varepsilon, X_{r}^{\varepsilon}\right) d r+\int_{0}^{t} \sigma\left(\omega, X_{r}^{\varepsilon} / \varepsilon, X_{r}^{\varepsilon}\right) d B_{r}$,

where $B$ is a standard d-dimensional Brownian motion and the parameter $\omega$ evolves in a random medium $\Omega$, that is a probability space with suitable stationarity and ergodicity properties. For each fixed value of the parameter $y \in \mathbb{R}^{d}$, the coefficients $b(\omega, \cdot, y), c(\omega, \cdot, y)$ and $\sigma(\omega, \cdot, y)$ are stationary random fields and for this reason are said to be locally stationary. The generator $\mathcal{L}^{\varepsilon}$ of the process $X^{\varepsilon}$ can be written in divergence form as

$$
\mathcal{L}^{\varepsilon}=(1 / 2) \sum_{i, j=1}^{d} \frac{\partial}{\partial x_{i}}\left([a+H](\omega, x / \varepsilon, x) \frac{\partial}{\partial x_{j}}\right)
$$

*Address: Ceremade, Université Paris-Dauphine, Place du maréchal De Lattre de Tassigny, 75775 Paris Cedex 16, France. Phone: (33)(0)1 440548 51. E-mail: rhodes@ceremade.dauphine.fr 
for an antisymmetric matrix $H$ and $a=\sigma \sigma^{*}$.

Roughly speaking, these diffusions stand for particles moving in a medium with two evolution scales: particles encounter smooth macroscopic variations, represented by the variable $x$, and fast microscopic ones, represented by the variable $x / \varepsilon$. It is a quite natural assumption that occurs in many modeling problems. That is the reason why, since the end of the sixties, this problem has been subject to many works, both by means of analytical tools (see [2] among others) or probabilistic tools (see [1]), in the locally periodic setting, that is when the matrices $a$ or $H$ are periodic with respect to the variable $x / \varepsilon$.

Thereafter, random media were introduced and homogenization of diffusions in random medium have been widely studied in the case when the random medium possesses microscopic variations only (cf. [4], [8], [9], and many others). However, as far as we know, the first attempt to generalize the results obtained in the locally periodic setting to the locally stationary one is due to Olla and Siri [12] (in a slightly more general framework). But the authors restrict themselves to the dimension 1 for technical reasons.

Let us try to briefly explain. If we look at a functional of the type

$$
\int_{0}^{t} f\left(\omega, X_{r}^{\varepsilon} / \varepsilon, X_{r}^{\varepsilon}\right) d r
$$

for some locally stationary random field $f$, its asymptotic behavior, as $\varepsilon$ goes to 0 , corresponds to a very intuitive argument. At a small scale, the oscillations due to the smallness of the parameter $\varepsilon$ make the functional average with respect to its first variable thanks to suitable ergodicity assumptions. In other words, this functional behaves, as $\varepsilon$ goes to 0 , as

$$
\int_{0}^{t} \bar{f}\left(X_{r}^{\varepsilon}\right) d r
$$

where $\bar{f}(y)$ is the mean over the medium of the random function $f(\omega, x, y)$. As guessed by the reader, this approximation is the key tool to pass to the limit in (1). This technique has been already used in the case of locally periodic coefficients. As explained in the already existing literature on this topic, the main difficulty actually lies in getting rid of the highly oscillating term $\frac{1}{\varepsilon} \int_{0}^{t} b\left(\omega, X_{r}^{\varepsilon} / \varepsilon, X_{r}^{\varepsilon}\right) d r$. The method consists in constructing the so-called correctors, that is solutions of auxiliary problems stated on the random medium. They have to make up for the microscopic oscillations of this functional.

From this remark on, our approach differs from Olla and Siri [12]. Their method consists in adapting the technics of the (non locally) stationary random setting. To sum up, they solve the equation $\mathcal{L}^{\varepsilon} u_{\varepsilon}=\left[\varepsilon^{-1} b+c\right](\omega, \cdot / \varepsilon, \cdot)$ and then tackle the description of the asymptotic behavior, as $\varepsilon$ tends to 0 of the solution $u_{\varepsilon}$. However, because of interactions between the microscopic and macroscopic scales, this description seems hard to carry through. To get 
round this difficulty, they choose the dimension as equal to one. Indeed, in this case only, they are provided with an explicit formula for the solution $u_{\varepsilon}$, expressed in terms of the coefficients. This formula is essential to conclude their work.

Our work is based on a separation of the microscopic and macroscopic scales, as already used in the locally periodic setting. But new difficulties arise due to the particular geometry/topology of a random medium in comparison with the torus (in the case of locally periodic coefficients). Let us develop this point. To construct the correctors, we make the following observation. From the microscopic point of view, the slowly varying macroscopic evolution of the medium can be seen as frozen. So the correctors are constructed by fixing the macroscopic evolution: for a fixed parameter $y \in \mathbb{R}^{d}$, they are defined as the (parameterized) solution $u(\omega, \cdot, y)$ of the equation

$$
(1 / 2) \sum_{i, j=1}^{d} \frac{\partial}{\partial x_{i}}\left([a+H](\omega, x, y) \frac{\partial}{\partial x_{j}} u(\omega, x, y)\right)=b(\omega, x, y), \quad x \in \mathbb{R}^{d}
$$

Applying the Itô formula then provides us with the following decomposition of the process

$$
\begin{aligned}
d X_{t}^{\varepsilon}+\varepsilon d u\left(\omega, X_{t}^{\varepsilon} / \varepsilon, X_{t}^{\varepsilon}\right) & =b \partial_{y} u\left(\omega, X_{t}^{\varepsilon} / \varepsilon, X_{t}^{\varepsilon}\right) d t+D\left(\omega, X_{t}^{\varepsilon} / \varepsilon, X_{t}^{\varepsilon}\right) d t \\
& +\left(\sigma+\partial_{x} u^{*} \sigma\right)\left(\omega, X_{t}^{\varepsilon} / \varepsilon, X_{t}^{\varepsilon}\right) d B_{t}+d R_{t}^{\varepsilon},
\end{aligned}
$$

where the process $R^{\varepsilon}$ reasonably converges to 0 a $\varepsilon$ tends to 0 and the function $D$ is a locally stationary random field. The main advantage of the correctors is that their contribution is small, that is $\varepsilon u\left(\omega, X_{t}^{\varepsilon} / \varepsilon, X_{t}^{\varepsilon}\right)$ converges to 0 as $\varepsilon$ goes to 0 , but they permit to remove the highly oscillating term from (6). In the locally periodic setting, the proof of the homogenization property is almost done. Indeed, in this context, it is possible to find a locally periodic solution $u$ of (5). As a consequence, the gradients $\partial_{x} u$ and $\partial_{y} u$ are also locally stationary and the approximation of (3) by (4) holds for each corresponding term in (6). The limit of $X^{\varepsilon}$ is then easily identified as the solution $X$ of the following SDE with some deterministic coefficients $A, B$

$$
X_{t}=x+\int_{0}^{t} B\left(X_{r}\right) d r+\int_{0}^{t} A\left(X_{r}\right) d B_{r} .
$$

However, the random context raises additional issues. Indeed, it is possible to find a solution $u$ of (5) with a locally stationary gradient $\partial_{x} u$ but it turns out that $u$ is not locally stationary. Thus, the term $\partial_{y} u$ is not locally stationary either in such a way that the approximation of (3) by (4) fails for the term $\int_{0}^{t} b \partial_{y} u\left(\omega, X_{r}^{\varepsilon} / \varepsilon, X_{r}^{\varepsilon}\right) d r$. This is the main issue of this paper. Up to introducing a new type of correctors for the function $b \partial_{y} u$, we prove that it can be splitted up into two parts: a locally stationary function $C$ and a second part $E$. This 
latter part can be approximated by a family $\left(E_{\varepsilon}\right)_{\varepsilon}$, which may be divergent in the $L^{2}$ sense but asymptotically not seen by the process $X^{\varepsilon}$, that is

$$
\int_{0}^{t} E_{\varepsilon}\left(\omega, X_{r}^{\varepsilon} / \varepsilon, X_{r}^{\varepsilon}\right) d r \rightarrow 0, \quad \text { as } \varepsilon \text { goes to } 0 .
$$

This convergence is established provided that we can control a sort of "Poincaré inequality" of the functions $\left(E_{\varepsilon}\right)_{\varepsilon}$, which connects both microscopic and macroscopic variations of the medium. This connection is of the utmost importance. But for it, corrections at a small scale would turn out to cause undesirable effects at a large scale and vice versa.

We are then in position to pass to the limit in (1) and prove that the limit process solves (7) for some deterministic coefficients $A, B$. We should point out that our result is an annealed convergence result, that is our convergence result is not stated for each realization $\omega$ of the random medium but in probability with respect to the measure of the random medium.

The organization of the paper is the following. In Section 2, we set out precisely the framework, in particular we define the random medium and give a complete description of the coefficients involved in (1). Our main result is stated in Section 3. The correctors are constructed in Section 4 and Section 5 explains how to apply the Itô formula to the correctors. Ergodic theorems are stated in Section 6, in particular the approximation of (3) by (4) is given a rigorous sense and conditions for (8) to hold are explained. The new correctors for

$\int_{0}^{t} b \partial_{y} u\left(\omega, X_{r}^{\varepsilon} / \varepsilon, X_{r}^{\varepsilon}\right) d r$ are introduced in Section (7), which also describes how to pass to the limit in (1) and get (7).

\section{Setup and Assumptions}

Random medium. From now on, $d \geq 1$ is a fixed integer. Following [7], we introduce the following

Definition 2.1. Let $(\Omega, \mathcal{G}, \mu)$ be a probability space and $\left\{\tau_{x} ; x \in \mathbb{R}^{d}\right\}$ a group of measure preserving transformations acting ergodically on $\Omega$ :

1) $\forall A \in \mathcal{G}, \forall x \in \mathbb{R}^{d}, \mu\left(\tau_{x} A\right)=\mu(A)$,

2) If for any $x \in \mathbb{R}^{d} \tau_{x} A=A$, then $\mu(A)=0$ or 1 ,

3) For any measurable function $\boldsymbol{g}$ on $(\Omega, \mathcal{G}, \mu)$, the function $(x, \omega) \mapsto \boldsymbol{g}\left(\tau_{x} \omega\right)$ is measurable on $\left(\mathbb{R}^{d} \times \Omega, \mathcal{B}\left(\mathbb{R}^{d}\right) \otimes \mathcal{G}\right)$.

The expectation with respect to the random medium is denoted by $\mathbb{M}$. Denote by $L^{2}(\Omega)$ the space of square integrable functions, by $|.|_{2}$ the corresponding norm and by $(., .)_{2}$ the associated inner product. The operators defined on $L^{2}(\Omega)$ by $T_{x} \boldsymbol{f}(\omega)=\boldsymbol{f}\left(\tau_{x} \omega\right)$ form a 
strongly continuous group of unitary maps in $L^{2}(\Omega)$. For every function $\boldsymbol{f} \in L^{2}(\Omega)$, let $f(\omega, x)=\boldsymbol{f}\left(\tau_{x} \omega\right)$. Each function $\boldsymbol{f}$ in $L^{2}(\Omega)$ defines in this way a stationary ergodic random field on $\mathbb{R}^{d}$. In what follows we will use the bold type to denote an element $\boldsymbol{f} \in L^{2}(\Omega)$ and the normal type $f(\omega, x)$ (or even $f(x)$ ) to distinguish from the associated stationary field. The group possesses $d$ generators (throughout this paper, $e_{i}$ stands for the i-th vector of the canonical basis of $\mathbb{R}^{d}$ )

$$
D_{i} \boldsymbol{g}=\lim _{h \rightarrow 0} \frac{T_{h e_{i}} \boldsymbol{g}-\boldsymbol{g}}{h} \text { if exists, }
$$

which are closed and densely defined. Setting

$$
\mathcal{C}=\operatorname{Span}\left\{\boldsymbol{g} \star \varphi ; \boldsymbol{g} \in L^{\infty}(\Omega), \varphi \in C_{c}^{\infty}\left(\mathbb{R}^{d}\right)\right\}, \text { with } \boldsymbol{g} \star \varphi(\omega)=\int_{\mathbb{R}^{d}} \boldsymbol{g}\left(\tau_{x} \omega\right) \varphi(x) d x
$$

the space $\mathcal{C}$ is dense in $L^{2}(\Omega)$ and $\mathcal{C} \subset \operatorname{Dom}\left(D_{i}\right)$ for all $1 \leq i \leq d$, with $D_{i}(\boldsymbol{g} \star \varphi)=$ $-\boldsymbol{g} \star \partial \varphi / \partial x_{i}$. If $\boldsymbol{g} \in \operatorname{Dom}\left(D_{i}\right)$, we also have $D_{i}(\boldsymbol{g} \star \varphi)=D_{i} \boldsymbol{g} \star \varphi$. For $\boldsymbol{f} \in \bigcap_{i=1}^{d} \operatorname{Dom}\left(D_{i}\right)$, we define the divergence operator Div by $\operatorname{Div} \boldsymbol{f}=\sum_{i=1}^{d} D_{i} \boldsymbol{f}$. We distinguish this latter operator from the usual divergence operator on $\mathbb{R}^{d}$ denoted by the small type div.

Locally stationary random fields. Following the notations introduced just above, for a measurable function $\boldsymbol{f}: \Omega \times \mathbb{R}^{d} \rightarrow \mathbb{R}^{n},(n \geq 1)$, we can consider the associated locally stationary random field $(x, y) \mapsto \boldsymbol{f}\left(\tau_{x} \omega, y\right)=f(\omega, x, y)$ (or even $f(x, y)$ ).

Structure of the coefficients. The coefficients $\boldsymbol{\sigma}: \Omega \times \mathbb{R}^{d} \rightarrow \mathbb{R}^{d \times d}, \boldsymbol{H}: \Omega \times \mathbb{R}^{d} \rightarrow \mathbb{R}^{d \times d}$ denote measurable functions with respect to the underlying product $\sigma$-fields and thus define locally stationary random fields. $\boldsymbol{H}$ is antisymmetric and $\boldsymbol{a}=\boldsymbol{\sigma} \boldsymbol{\sigma}^{*}$. Furthermore, for some positive constant $\Lambda$ (independent of $\omega$ ), $\boldsymbol{\sigma}$ and $\boldsymbol{H}$ satisfy

Assumption 2.2. (Regularity). For each fixed $\omega \in \Omega$, the coefficients $\sigma(\omega, .,$.$) and H(\omega, .,$. are two times continuously differentiable with respect to each variable and are, as well as their derivatives up to order two, $\Lambda$-Lipschitzian and bounded by $\Lambda$.

\section{Assumption 2.3. (Uniform ellipticity)}

There exists a constant $M$ such that the matrix-valued function $\boldsymbol{a}(\omega, y)=\boldsymbol{\sigma} \boldsymbol{\sigma}^{*}(\omega, y)$ satisfies for every $y \in \mathbb{R}^{d}$

$$
M^{-1} \mathrm{I} \leq \boldsymbol{a}(., y) \leq M \mathrm{I},
$$

where I stands for the $d \times d$ identity matrix.

Remark. Assumptions 2.2 may appear restrictive and can surely be relaxed (see [3] for results in this direction). In particular, the statement of the homogenization property only involves the 
derivatives of order 1 with respect to $y \in \mathbb{R}^{d}$ (see Theorem 3.1). However, it avoids dealing with heavy regularizing procedure that are not the purpose of this work.

Diffusion in a locally stationary random environment. For $j=1, \ldots, d$, we define the coefficients

$$
\boldsymbol{b}_{j}(\omega, y)=\frac{1}{2} \sum_{i=1}^{d} D_{i}(\boldsymbol{a}+\boldsymbol{H})_{i j}(\omega, y), \quad \boldsymbol{c}_{j}(\omega, y)=\frac{1}{2} \sum_{i=1}^{d} \partial_{y_{i}}(\boldsymbol{a}+\boldsymbol{H})_{i j}(\omega, y) .
$$

From Assumption 2.2, the coefficients $b_{j}(\omega, .,$.$) and c_{j}(\omega, .,$.$) are Lipschitzian so that, for a$ starting point $x \in \mathbb{R}^{d}$ and $\varepsilon>0$, we can consider the strong solution $X^{\varepsilon}$ of the following SDE with locally stationary coefficients:

$$
X_{t}^{\varepsilon}=x+\frac{1}{\varepsilon} \int_{0}^{t} b\left(\bar{X}_{r}^{\varepsilon}, X_{r}^{\varepsilon}\right) d r+\int_{0}^{t} c\left(\bar{X}_{r}^{\varepsilon}, X_{r}^{\varepsilon}\right) d r+\int_{0}^{t} \sigma\left(\bar{X}_{r}^{\varepsilon}, X_{r}^{\varepsilon}\right) d B_{r}
$$

where we have set $\bar{X}_{t}^{\varepsilon} \equiv X_{t}^{\varepsilon} / \varepsilon$ and $B$ is a standard d-dimensional Brownian motion. We should point out that the generator of this diffusion could be written in divergence form as

$$
\mathcal{L}^{\varepsilon}=(1 / 2) \sum_{i, j=1}^{d} \frac{\partial}{\partial x_{i}}\left([a+H](\omega, x / \varepsilon, x) \frac{\partial}{\partial x_{j}}\right) .
$$

Notations. Note that the law of the process $X^{\varepsilon}$ depends on $\omega$ even if this parameter does not appear in the notation $X^{\varepsilon}$. For the sake of simplicity, we indicate the starting point $x$ of $X^{\varepsilon}$ by writing, when necessary, $\mathbb{P}_{x}^{\varepsilon}$ (and $\mathbb{E}_{x}^{\varepsilon}$ for the corresponding expectation), this avoids heavy notations as $X^{\varepsilon, x}$. We can then consider the probability measure $\overline{\mathbb{P}}_{x}^{\varepsilon} \equiv \mathbb{M}\left[\mathbb{P}_{x}^{\varepsilon}().\right]$ (the so-called annealed law of the process $X^{\varepsilon}$ ) and its expectation $\overline{\mathbb{E}}_{x}^{\varepsilon}$. In the sequel, the generic notations " $C$ " and " $D$ " stand for constants that only refer to $M$ and $\Lambda$. Dependencies on additional parameters are always mentioned.

\section{Main Results}

We are now in position to state the main result of this paper.

Theorem 3.1. Homogenization. For each fixed $x \in \mathbb{R}^{d}$, the law of the process $X^{\varepsilon}$ converges in $C\left([0, T] ; \mathbb{R}^{d}\right)$, in probability with respect to $\mu$, towards the law of the process $X$ that solves the following SDE with deterministic coefficients (they do not depend on the medium $\Omega$ ):

$$
X_{t}=x+\int_{0}^{t} \bar{B}\left(X_{r}\right) d r+\int_{0}^{t} \bar{A}^{1 / 2}\left(X_{r}\right) d B_{r}
$$


The coefficients $\bar{A}$ and $\bar{B}$ are of class $C^{2}$ and are defined, for $y \in \mathbb{R}^{d}$, by

$$
\begin{aligned}
& \bar{A}(y)=\lim _{\lambda \rightarrow 0} \mathbb{M}\left[\left(I+\boldsymbol{D} \boldsymbol{u}_{\lambda}\right)^{*} \boldsymbol{a}\left(I+\boldsymbol{D} \boldsymbol{u}_{\lambda}\right)(., y)\right], \\
& \bar{H}(y)=\lim _{\lambda \rightarrow 0} \mathbb{M}\left[\left(I+\boldsymbol{D} \boldsymbol{u}_{\lambda}\right)^{*} \boldsymbol{H}\left(I+\boldsymbol{D} \boldsymbol{u}_{\lambda}\right)(., y)\right], \\
& \bar{B}(y)=(1 / 2) \partial_{y}(\bar{A}+\bar{H})(y)
\end{aligned}
$$

Formally speaking, for each $y \in \mathbb{R}^{d}$ and $\lambda>0$, the entries $\left(\boldsymbol{u}_{\lambda}^{i}(., y)\right)_{1 \leq i \leq d}$ of the function $\boldsymbol{u}_{\lambda}(., y): \Omega \rightarrow \mathbb{R}^{d}$ solve the following so-called auxiliary problems, which are stated on the random medium

$$
\lambda \boldsymbol{u}_{\lambda}^{i}(., y)-\frac{1}{2} \sum_{j, k} D_{j}\left[\left(\boldsymbol{a}_{j k}+\boldsymbol{H}_{j k}\right) D_{k} \boldsymbol{u}_{\lambda}^{i}(., y)\right]=\boldsymbol{b}_{i}(., y) .
$$

A rigorous description of $\boldsymbol{u}_{\lambda}(., y)$ is given in Section 4.

Remark 1. Since the matrix $\boldsymbol{a}$ is uniformly elliptic, the homogenized coefficient $\bar{A}$ also is. Indeed, because of the stationarity of the measure $\mu$, we have $\mathbb{M}\left[D \boldsymbol{u}_{\lambda}(., y)\right]=0$. Then, for any $X \in \mathbb{R}^{d}$ and $y \in \mathbb{R}^{d}$, it is readily seen that

$$
|X|^{2} \leq \mathbb{M}\left[\left|X+X \cdot D \boldsymbol{u}_{\lambda}(., y)\right|^{2}\right] \leq M X^{*} \mathbb{M}\left[\left(\mathrm{I}+D \boldsymbol{u}_{\lambda}\right)^{*} \boldsymbol{a}\left(\mathrm{I}+D \boldsymbol{u}_{\lambda}\right)(., y)\right] X \text {. }
$$

By passing to the limit as $\lambda \rightarrow 0$ in the above expression and using (15a), the uniform ellipticity of $\bar{A}$ follows.

Remark 2. As mentioned in Section 1, our result is an annealed convergence result. The reader may wonder if a quenched result holds. In other words, can we establish that the law of the process $X^{\varepsilon}$ converges for (almost) every fixed realization $\omega$ of the random medium? The main difficulty to prove such a result actually lies in establishing a quenched version of Theorem 6.3.

\section{Auxiliary Problems}

Setup and notations. Let us now introduce the different tools we will use on the medium. We aim at extending the following unbounded operators on $L^{2}(\Omega)$ defined on $\mathcal{C}$ by

$$
\boldsymbol{S}^{y} \equiv(1 / 2) \sum_{i, j=1}^{d} D_{i}\left(\boldsymbol{a}_{i j}(\cdot, y) D_{j} \cdot\right), \quad \boldsymbol{L}^{y} \equiv(1 / 2) \sum_{i, j=1}^{d} D_{i}\left([\boldsymbol{a}+\boldsymbol{H}]_{i j}(., y) D_{j} \cdot\right)
$$


by following the construction of $[5$, Ch. 3, Sect 3.] or [10, Ch. 1, Sect 2.]. For any $\varphi, \psi \in \mathcal{C}$, we define

$$
\begin{aligned}
(\boldsymbol{\varphi}, \boldsymbol{\psi})_{1, y} & \equiv-(\boldsymbol{\varphi}, \boldsymbol{S}(\cdot, y) \boldsymbol{\psi})_{2}=(1 / 2)(\boldsymbol{a}(\cdot, y) D \boldsymbol{\varphi}, D \boldsymbol{\psi})_{2} \\
(\boldsymbol{\varphi}, \boldsymbol{\psi})_{1} & \equiv(1 / 2)(D \boldsymbol{\varphi}, D \boldsymbol{\psi})_{2}
\end{aligned}
$$

and the associated seminorms $\|\varphi\|_{1, y}^{2} \equiv(\boldsymbol{\varphi}, \boldsymbol{\varphi})_{1, y}$ and $\|\boldsymbol{\varphi}\|_{1}^{2} \equiv(\boldsymbol{\varphi}, \boldsymbol{\varphi})_{1}$, which are all equivalent. Then we can define for any $\lambda>0$ and $\varphi, \psi \in \mathcal{C}$

$$
\mathcal{E}_{\lambda}^{y}(\boldsymbol{\varphi}, \boldsymbol{\psi}) \equiv \lambda(\boldsymbol{\varphi}, \boldsymbol{\psi})_{2}+(\boldsymbol{\varphi}, \boldsymbol{\psi})_{1, y}, \mathcal{E}(\boldsymbol{\varphi}, \boldsymbol{\psi}) \equiv(\boldsymbol{\varphi}, \boldsymbol{\psi})_{2}+(\boldsymbol{\varphi}, \boldsymbol{\psi})_{1}
$$

All these define inner products on $\mathcal{C} \times \mathcal{C}$ and we will denote by $\mathbb{H}_{1}$ the closure of $\mathcal{C}$ with respect to the resulting norms, which are equivalent too. For any $y \in \mathbb{R}^{d}$ and $\lambda>0$, we introduce a new (nonsymmetric) bilinear form on $\mathcal{C} \times \mathcal{C}$ by

$$
\mathcal{B}_{\lambda}^{y}(\boldsymbol{\varphi}, \boldsymbol{\psi})=\lambda(\boldsymbol{\varphi}, \boldsymbol{\psi})_{2}-\left(\boldsymbol{L}^{y} \boldsymbol{\varphi}, \boldsymbol{\psi}\right)_{2}
$$

From Assumptions 2.2 and 2.3, it is clearly continuous with respect to $\mathcal{E}$ so that it extends to $\mathbb{H}_{1} \times \mathbb{H}_{1}$ (this extension is still denoted $\mathcal{B}_{\lambda}^{y}$ ). Now, according to [5, Ch. 3, Sect 3.] and [10, Ch. 1, Sect 2.], we can extend $\boldsymbol{S}^{y}$ and $\boldsymbol{L}^{y}$ as follows. We define $\operatorname{Dom}\left(\boldsymbol{S}^{y}\right)=$ $\left\{\boldsymbol{\varphi} \in \mathbb{H}_{1} ; \mathcal{E}_{\lambda}^{y}(\boldsymbol{\varphi}, \cdot)\right.$ is $L^{2}$ continuous $\}$ and $\operatorname{Dom}\left(\boldsymbol{L}^{y}\right)=\left\{\mathcal{B}_{\lambda}^{y}(\boldsymbol{\varphi}, \cdot)\right.$ is $L^{2}$ continuous $\}$. For $\boldsymbol{\varphi} \in \operatorname{Dom}\left(\boldsymbol{S}^{y}\right)$ (resp. $\boldsymbol{\varphi} \in \operatorname{Dom}\left(\boldsymbol{L}^{y}\right)$ ), we can find $\boldsymbol{f} \in L^{2}(\Omega)$ such that $\mathcal{E}_{\lambda}^{y}(\boldsymbol{\varphi}, \cdot)=(\boldsymbol{f}, \cdot)_{2}$ (resp. $\left.\mathcal{B}_{\lambda}^{y}(\boldsymbol{\varphi}, \cdot)=(\boldsymbol{f}, \cdot)_{2}\right)$. Then $\boldsymbol{S}^{y} \boldsymbol{\varphi}\left(\right.$ resp. $\left.\boldsymbol{L}^{y} \boldsymbol{\varphi}\right)$ is defined as $\lambda \boldsymbol{\varphi}-\boldsymbol{f}($ resp. $\lambda \boldsymbol{\varphi}-\boldsymbol{f})$. It can be proved that this definition does not depend on $\lambda>0$. Moreover, $\boldsymbol{S}^{y}$ is selfadjoint and $\boldsymbol{L}^{y}=\left(\left(\boldsymbol{L}^{y}\right)^{*}\right)^{*}$, where the adjoint $\left(\boldsymbol{L}^{y}\right)^{*}$ of $\boldsymbol{L}^{y}$ can be described as follows: $\operatorname{Dom}\left(\left(\boldsymbol{L}^{y}\right)^{*}\right)=\left\{\boldsymbol{\varphi} \in \mathbb{H}_{1} ; \mathcal{B}_{\lambda}^{y}(\cdot, \boldsymbol{\varphi})\right.$ is $L^{2}$ continuous $\}$ and for $\boldsymbol{\varphi} \in \operatorname{Dom}\left(\left(\boldsymbol{L}^{y}\right)^{*}\right)$, we can find $\boldsymbol{f} \in L^{2}(\Omega)$ such that $\mathcal{B}_{\lambda}^{y}(\cdot, \boldsymbol{\varphi})=(\boldsymbol{f}, \cdot)_{2}$. Then $\left(\boldsymbol{L}^{y}\right)^{*} \boldsymbol{\varphi}$ exactly matches $\lambda \boldsymbol{\varphi}-\boldsymbol{f}$. Throughout this paper, we will widely use the following relation without referring to it anymore: for $y \in \mathbb{R}^{d}$ and $\boldsymbol{\varphi} \in \operatorname{Dom}\left(\boldsymbol{L}^{y}\right), \boldsymbol{\psi} \in \mathbb{H}_{1}$,

$$
\left(\boldsymbol{L}^{y} \boldsymbol{\varphi}, \boldsymbol{\psi}\right)_{2}=-\frac{1}{2}((\boldsymbol{a}+\boldsymbol{H})(\cdot, y) D \boldsymbol{\varphi}, D \boldsymbol{\psi})_{2} .
$$

If a function $\boldsymbol{b}$ satisfy the property:

$$
\exists C>0, \forall \boldsymbol{\varphi} \in \mathcal{C}, \quad(\boldsymbol{b}, \boldsymbol{\varphi})_{2} \leq C\|\boldsymbol{\varphi}\|_{1},
$$

then we will say that $\boldsymbol{b} \in \mathbb{H}_{-1}$ and we will define $\|\boldsymbol{b}\|_{-1}$ as the smallest constant $C$ that satisfies this property.

Finally, we define the space $\mathbb{D}$ as the closure in $\left(L^{2}(\Omega)\right)^{d}$ of the set $\{D \boldsymbol{\varphi} ; \boldsymbol{\varphi} \in \mathcal{C}\}$. By this way, each function $\varphi \in \mathbb{H}_{1}$ admits a "gradient" that we still denote by $D \varphi$. 
Solvability and Regularity of the Resolvent Equation. Fix $\lambda>0$ and $y \in \mathbb{R}^{d}$. From Assumption 2.3, the boundedness and the antisymmetry of $\boldsymbol{H}(., y)$, the bilinear form $\mathcal{B}_{\lambda}^{y}$ (see (18)) is clearly continuous and coercive on $\mathbb{H}_{1}$. It thus defines a resolvent operator $G_{\lambda}^{y}$ associated to the operator $\lambda-\boldsymbol{L}^{y}$. In particular, for $\boldsymbol{h} \in L^{2}(\Omega), \boldsymbol{u}_{\lambda}(., y) \equiv G_{\lambda}^{y} \boldsymbol{h}$ belongs to $\mathbb{H}_{1} \cap \operatorname{Dom}\left(\boldsymbol{L}^{y}\right)$ and satisfies $\lambda \boldsymbol{u}_{\lambda}(\cdot, y)-\boldsymbol{L}^{y} \boldsymbol{u}_{\lambda}(\cdot, y)=\boldsymbol{h}$.

We now investigate the regularity of $\boldsymbol{u}_{\lambda}(\cdot, y)$ with respect to the parameter $y$.

Proposition 4.1. Let us consider $\boldsymbol{h}: y \in \mathbb{R}^{d} \mapsto \boldsymbol{h}(., y) \in L^{2}(\Omega)$ and $\boldsymbol{f}: y \in \mathbb{R}^{d} \mapsto \boldsymbol{f}(., y) \in$ $L^{2}(\Omega) \cap \mathbb{H}_{-1}$. Suppose that there exist two constants $C_{2}, C_{-1}$ such that:

1) the application $y \mapsto \boldsymbol{h}(., y) \in L^{2}(\Omega)$ is two times continuously differentiable in $L^{2}(\Omega)$. The derivatives up to order 2 are bounded by $C_{2}$ in $L^{2}(\Omega)$ and are $C_{2}$-Lipschitz in $L^{2}(\Omega)$.

2) the application $y \mapsto \boldsymbol{f}(., y) \in L^{2}(\Omega) \cap \mathbb{H}_{-1}$ is two times continuously differentiable in $\mathbb{H}_{-1}$. The derivatives up to order 2 are bounded by $C_{-1}$ in $\mathbb{H}_{-1}$ and are $C_{-1}$-Lipschitz in $\mathbb{H}_{-1}$.

Then, for any $\lambda>0$, the solution $\boldsymbol{u}_{\lambda}(., y) \in \mathbb{H}_{1} \cap \operatorname{Dom}\left(\boldsymbol{L}^{y}\right)$ of the equation

$$
\lambda \boldsymbol{u}_{\lambda}(., y)-\boldsymbol{L}^{y} \boldsymbol{u}_{\lambda}(., y)=\boldsymbol{h}(., y)+\boldsymbol{f}(., y)
$$

is two times continuously differentiable in $\mathbb{H}_{1}$ with respect to the parameter $y \in \mathbb{R}^{d}$. Furthermore there exists a constant $D_{4.1}>0$, which only depends on $\Lambda, M, C_{-1}$, such that the functions $\boldsymbol{g}_{\lambda}(., y)=\boldsymbol{u}_{\lambda}(., y), \partial_{y} \boldsymbol{u}_{\lambda}(., y), \partial_{y y}^{2} \boldsymbol{u}_{\lambda}(., y)$ satisfy the property: $\forall(y, h) \in \mathbb{R}^{2}$,

$$
\begin{aligned}
& \lambda\left|\boldsymbol{g}_{\lambda}(., y)\right|_{2}^{2}+\left\|\boldsymbol{g}_{\lambda}(., y)\right\|_{1}^{2} \leq D_{4.1}\left(1+C_{2}^{2} / \lambda\right), \\
& \lambda\left|\boldsymbol{g}_{\lambda}(., y+h)-\boldsymbol{g}_{\lambda}(., y)\right|_{2}^{2}+\left\|\boldsymbol{g}_{\lambda}(., y+h)-\boldsymbol{g}_{\lambda}(., y)\right\|_{1}^{2} \leq D_{4.1}\left(1+C_{2}^{2} / \lambda\right)|h|^{2} .
\end{aligned}
$$

Proof: For every $\varphi \in \mathbb{H}_{1}$, (20) provides us with the following weak formulation

$$
\lambda\left(\boldsymbol{u}_{\lambda}(., y), \boldsymbol{\varphi}\right)_{2}+(1 / 2)\left([\boldsymbol{a}+\boldsymbol{H}] D \boldsymbol{u}_{\lambda}(., y), D \boldsymbol{\varphi}\right)_{2}=(\boldsymbol{h}(., y), \boldsymbol{\varphi})_{2}+(\boldsymbol{f}(., y), \boldsymbol{\varphi})_{-1,1} .
$$

Choosing $\varphi=\boldsymbol{u}_{\lambda}(., y)$, (22) yields

$$
\begin{aligned}
\lambda\left|\boldsymbol{u}_{\lambda}(., y)\right|_{2}^{2}+M^{-1}\left\|\boldsymbol{u}_{\lambda}(., y)\right\|_{1}^{2} & \leq|\boldsymbol{h}(., y)|_{2}\left|\boldsymbol{u}_{\lambda}(., y)\right|_{2}+\|\boldsymbol{f}(., y)\|_{-1}\left\|\boldsymbol{u}_{\lambda}(., y)\right\|_{1} \\
& \leq \frac{|\boldsymbol{h}(., y)|_{2}^{2}}{2 \lambda}+\frac{\lambda\left|\boldsymbol{u}_{\lambda}(., y)\right|_{2}^{2}}{2}+M \frac{\|\boldsymbol{f}(., y)\|_{-1}^{2}}{2}+\frac{\left\|\boldsymbol{u}_{\lambda}(., y)\right\|_{1}^{2}}{2 M}
\end{aligned}
$$

from which we derive $\lambda\left|\boldsymbol{u}_{\lambda}(., y)\right|_{2}^{2}+M^{-1}\left\|\boldsymbol{u}_{\lambda}(., y)\right\|_{1}^{2} \leq \lambda^{-1} C_{2}^{2}+M C_{-1}^{2}$.

Let us investigate now the difference $\boldsymbol{v}_{\lambda}(., y, h)=\boldsymbol{u}_{\lambda}(., y+h)-\boldsymbol{u}_{\lambda}(., y)$, for $y, h \in \mathbb{R}^{d}$.

Thanks to (22), we have for any $\varphi \in \mathbb{H}_{1}$

$$
\begin{aligned}
\lambda & \left(\boldsymbol{v}_{\lambda}(., y, h), \boldsymbol{\varphi}\right)_{2}+(1 / 2)\left([\boldsymbol{a}+\boldsymbol{H}](\cdot, y) D \boldsymbol{v}_{\lambda}(., y, h), D \boldsymbol{\varphi}\right)_{2} \\
= & (\boldsymbol{h}(., y+h)-\boldsymbol{h}(., y), \boldsymbol{\varphi})_{2}+(\boldsymbol{f}(., y+h)-\boldsymbol{f}(., y), \boldsymbol{\varphi})_{-1,1} \\
& +(1 / 2)\left([\boldsymbol{a}(., y)-\boldsymbol{a}(., y+h)+\boldsymbol{H}(., y)-\boldsymbol{H}(., y+h)] D \boldsymbol{u}_{\lambda}(., y+h), D \boldsymbol{\varphi}\right)_{2} .
\end{aligned}
$$


From the Lipschitz assumptions on the coefficients, for any $\boldsymbol{\varphi}, \boldsymbol{\psi} \in \mathbb{H}_{1}$,

$$
(1 / 2)([\boldsymbol{a}(., y)-\boldsymbol{a}(., y+h)+\boldsymbol{H}(., y)-\boldsymbol{H}(., y+h)] D \boldsymbol{\varphi}, D \boldsymbol{\psi}) \leq \Lambda|h|\|\boldsymbol{\varphi}\|_{1}\|\boldsymbol{\psi}\|_{1} .
$$

Choosing $\boldsymbol{\varphi}=\boldsymbol{v}_{\lambda}(., y, h)$ in (23) then leads to (in what follows, $C\left(M, \Lambda, C_{-1}\right)$ stands for a constant that only depends on $M, \Lambda$ and $C_{-1}$ )

$$
\begin{aligned}
\lambda \mid \boldsymbol{v}_{\lambda}(., y, h) & \left.\right|_{2} ^{2}+M^{-1}\left\|\boldsymbol{v}_{\lambda}(., y, h)\right\|_{1}^{2} \\
& \leq C_{2}\left|h\left\|\left.\boldsymbol{v}_{\lambda}(., y, h)\right|_{2}+C_{-1}|h|\right\| \boldsymbol{v}_{\lambda}(., y, h)\left\|_{1}+\Lambda|h|\right\| \boldsymbol{u}_{\lambda}(., y+h)\left\|_{1}\right\| \boldsymbol{v}_{\lambda}(., y, h) \|_{1}\right. \\
& \leq C_{2}\left|h\left\|\left.\boldsymbol{v}_{\lambda}(., y, h)\right|_{2}+|h|\left(\Lambda\left\|\boldsymbol{u}_{\lambda}(., y+h)\right\|_{1}+C_{-1}\right)\right\| \boldsymbol{v}_{\lambda}(., y, h) \|_{1}\right. \\
& \leq(\lambda / 2)\left|\boldsymbol{v}_{\lambda}(., y, h)\right|_{2}+(1 / 2 M)\left\|\boldsymbol{v}_{\lambda}(., y, h)\right\|_{1}^{2}+|h|^{2} C\left(M, \Lambda, C_{-1}\right)\left(C_{2}^{2} / \lambda+1\right)
\end{aligned}
$$

and finally gives us

$$
\lambda\left|\boldsymbol{v}_{\lambda}(., y, h)\right|_{2}^{2}+M^{-1}\left\|\boldsymbol{v}_{\lambda}(., y, h)\right\|_{1}^{2} \leq|h|^{2} C\left(M, \Lambda, C_{-1}\right)\left(C_{2}^{2} / \lambda+1\right) .
$$

So, for each $i=1, \ldots, d$ (remind that $e_{i}$ denotes the $\mathrm{i}$-th vector of the canonical basis of $\mathbb{R}^{d}$ ) and $h \in \mathbb{R}$, the family $\left(h^{-1}\left[\boldsymbol{u}_{\lambda}\left(., y+h e_{i}\right)-\boldsymbol{u}_{\lambda}(., y)\right]\right)_{h \in \mathbb{R}}$ is bounded in the space $\mathbb{H}_{1}$ so that this family is weakly compact in $\mathbb{H}_{1}$. Let $\boldsymbol{w}_{i}(., y)$ be the limit of a converging subsequence. From (23), it is plain to see that this limit is actually the unique weak solution of the equation

$$
\begin{aligned}
\lambda\left(\boldsymbol{w}_{i}(., y), \boldsymbol{\varphi}\right)_{2}+(1 / 2)\left([\boldsymbol{a}+\boldsymbol{H}](., y) D \boldsymbol{w}_{i}(., y), D \boldsymbol{\varphi}\right)_{2} \\
=\left(\partial_{y_{i}} \boldsymbol{h}(., y), \boldsymbol{\varphi}\right)_{2}+\left(\partial_{y_{i}} \boldsymbol{f}(., y), \boldsymbol{\varphi}\right)_{-1,1}+(1 / 2)\left(\partial_{y_{i}}[\boldsymbol{a}+\boldsymbol{H}](., y) D \boldsymbol{u}_{\lambda}(., y), D \boldsymbol{\varphi}\right)_{2} .
\end{aligned}
$$

Subtracting this latter expression from $h^{-1} \times(23)$ and choosing $\varphi=h^{-1} \boldsymbol{v}\left(\cdot, y, h e_{i}\right)-\boldsymbol{w}_{i}(., y)$, we can prove that $h^{-1}\left[\boldsymbol{u}_{\lambda}\left(., y+h e_{i}\right)-\boldsymbol{u}_{\lambda}(., y)\right] \rightarrow \boldsymbol{w}_{i}$ in $\mathbb{H}_{1}$ as $h$ tends to 0 . The same job can be carried out for the second order derivatives.

Auxiliary problems. Let us now tackle the study of the so-called auxiliary problems. For $i=1, \ldots, d$, let us consider the solution $\boldsymbol{u}_{\lambda}^{i}(., y)$ of the equation

$$
\lambda \boldsymbol{u}_{\lambda}^{i}(., y)-\boldsymbol{L}^{y} \boldsymbol{u}_{\lambda}^{i}(., y)=\boldsymbol{b}_{i}(., y),
$$

where $\boldsymbol{b}_{i}(., y)=(1 / 2) \sum_{k=1}^{d} D_{k}(\boldsymbol{a}+\boldsymbol{H})_{k i}(., y)$ (see (11)). The weak form of the resolvent equation (24) then reads for $\varphi \in \mathcal{C}$

$$
\lambda\left(\boldsymbol{u}_{\lambda}^{i}(., y), \boldsymbol{\varphi}\right)_{2}+(1 / 2)\left([\boldsymbol{a}+\boldsymbol{H}] D \boldsymbol{u}_{\lambda}^{i}(., y), D \boldsymbol{\varphi}\right)_{2}=-(1 / 2)\left([\boldsymbol{a}+\boldsymbol{H}](., y) e_{i}, D \boldsymbol{\varphi}\right)_{2} .
$$

Lemma 4.2. The mapping $y \mapsto \boldsymbol{b}_{i}(., y) \in L^{2}(\Omega) \cap \mathbb{H}_{-1}$ is two times continuously differentiable in $\mathbb{H}_{-1}$ with bounded Lipschitzian derivatives in $\mathbb{H}_{-1}$. 
Proof: First note that $\boldsymbol{b}_{i}(., y) \in \mathbb{H}_{-1}$. Indeed, for each $\varphi \in \mathcal{C}$, from Assumptions 2.2 and 2.3,

$$
\left(\boldsymbol{b}_{i}(., y), \boldsymbol{\varphi}\right)_{2}=-(1 / 2)\left((\boldsymbol{a}+\boldsymbol{H})(., y) e_{i}, D \boldsymbol{\varphi}\right)_{2} \leq(M+\Lambda)\|\boldsymbol{\varphi}\|_{1} .
$$

From Assumptions 2.2, it is readily seen that the $\mathbb{H}_{-1}$ derivatives of $\boldsymbol{b}_{i}$ coincide, for $1 \leq k \leq d$, with the classical derivatives $\partial_{y_{k}} \boldsymbol{b}_{i}$ and

$$
\left(\partial_{y_{k}} \boldsymbol{b}_{i}(., y), \boldsymbol{\varphi}\right)_{2}=-(1 / 2)\left(\left(\partial_{y_{k}} \boldsymbol{a}+\partial_{y_{k}} \boldsymbol{H}\right)(., y) e_{i}, D \boldsymbol{\varphi}\right)_{2} \leq \Lambda\|\boldsymbol{\varphi}\|_{1} .
$$

The same job can be carried out for the second order derivatives. Details are left to the reader.

From Proposition 4.1 (with the functions $\boldsymbol{h}=0$ and $\boldsymbol{f}=\boldsymbol{b}_{i}$ ), the function $y \mapsto \boldsymbol{u}_{\lambda}^{i}(., y)$ is two times continuously differentiable in $\mathbb{H}_{1}$. We now concentrate our efforts on describing the asymptotic behavior of $\boldsymbol{u}_{\lambda}^{i}$, as well as its derivatives, as $\lambda$ goes to 0 .

Proposition 4.3. For each fixed $y \in \mathbb{R}^{d}$ and $1 \leq i \leq d$, the family $\left(D \boldsymbol{u}_{\lambda}^{i}(., y)\right)_{\lambda}$ converges to a limit $\boldsymbol{\xi}_{i}(., y) \in L^{2}(\Omega)^{d}$ as $\lambda$ goes to 0 . The same property holds for the derivatives, that is, the families $\left(D \partial_{y_{j}} \boldsymbol{u}_{\lambda}^{i}(., y)\right)_{\lambda},\left(D \partial_{y_{j} y_{k}}^{2} \boldsymbol{u}_{\lambda}^{i}(., y)\right)_{\lambda}(1 \leq i, j, k \leq d)$ respectively converge to $\partial_{y_{j}} \boldsymbol{\xi}_{i}(., y), \partial_{y_{j} y_{k}}^{2} \boldsymbol{\xi}_{i}(., y)$ in $L^{2}(\Omega)^{d}$. Furthermore, we have

$$
\lambda\left|\boldsymbol{u}_{\lambda}^{i}(., y)\right|_{2}^{2}+\lambda\left|\partial_{y_{j}} \boldsymbol{u}_{\lambda}^{i}(., y)\right|_{2}^{2}+\lambda\left|\partial_{y_{j} y_{k}}^{2} \boldsymbol{u}_{\lambda}^{i}(., y)\right|_{2}^{2} \rightarrow 0, \quad \text { as } \lambda \text { tends to } 0,
$$

and, each function $\boldsymbol{g}_{\lambda}(., y)=\boldsymbol{u}_{\lambda}^{i}(., y), \partial_{y_{k}} \boldsymbol{u}_{\lambda}^{i}(., y), \partial_{y_{k} y_{l}} \boldsymbol{u}_{\lambda}^{i}(., y)$ satisfies the property:

$$
\lambda\left|\boldsymbol{g}_{\lambda}(., y)\right|_{2}^{2}+\left\|\boldsymbol{g}_{\lambda}(., y)\right\|_{1}^{2} \leq C_{4.3}
$$

$$
\lambda\left|\boldsymbol{g}_{\lambda}(., y+h)-\boldsymbol{g}_{\lambda}(., y)\right|_{2}^{2}+\left\|\boldsymbol{g}_{\lambda}(., y+h)-\boldsymbol{g}_{\lambda}(., y)\right\|_{1}^{2} \leq C_{4.3}|h|^{2}
$$

for every $y, h \in \mathbb{R}^{d}$, where $C_{4.3}$ is a positive constant independent of $\lambda>0$ and $y \in \mathbb{R}^{d}$.

Proof: From Lemma 4.2, Proposition 4.1 applies with $\boldsymbol{h}=0$ and $\boldsymbol{f}=\boldsymbol{b}_{i}$ and provides us with the estimate $\lambda\left|\boldsymbol{u}_{\lambda}^{i}(., y)\right|_{2}^{2}+\left|D \boldsymbol{u}_{\lambda}^{i}(., y)\right|_{2}^{2} \leq C$. Let us denote by $\boldsymbol{\xi}_{i}(., y) \in L^{2}(\Omega)^{d}$ a weak limit of the family $\left(D \boldsymbol{u}_{\lambda}^{i}(., y)\right)_{\lambda}$ as $\lambda$ goes to 0 . By passing to the limit in (25), it is plain to see that $\forall \varphi \in \mathcal{C}$

$$
(1 / 2)\left([\boldsymbol{a}+\boldsymbol{H}] \boldsymbol{\xi}_{i}(., y), D \boldsymbol{\varphi}\right)_{2}=-(1 / 2)\left([\boldsymbol{a}+\boldsymbol{H}](., y) e_{i}, D \boldsymbol{\varphi}\right)_{2} .
$$

The limit is clearly unique in $\mathbb{D}$ because $(\boldsymbol{\chi}, \boldsymbol{\phi}) \in \mathbb{D} \times \mathbb{D} \mapsto([\boldsymbol{a}+\boldsymbol{H}] \boldsymbol{\chi}, \boldsymbol{\phi})_{2}$ is coercive on $\mathbb{D} \times \mathbb{D}$. Comparing (25) and (27) yields

$$
\lambda\left(\boldsymbol{u}_{\lambda}^{i}(., y), \boldsymbol{\varphi}\right)_{2}+(1 / 2)\left([\boldsymbol{a}+\boldsymbol{H}] D \boldsymbol{u}_{\lambda}^{i}(., y), D \boldsymbol{\varphi}\right)_{2}=(1 / 2)\left([\boldsymbol{a}+\boldsymbol{H}] \boldsymbol{\xi}_{i}(., y), D \boldsymbol{\varphi}\right)_{2} .
$$


We choose $\boldsymbol{u}_{\lambda}^{i}(., y)=\boldsymbol{\varphi}$, so that the Cauchy-Schwarz inequality yields:

$\lambda\left|\boldsymbol{u}_{\lambda}^{i}(., y)\right|_{2}^{2}+(1 / 2)\left([\boldsymbol{a}+\boldsymbol{H}] D \boldsymbol{u}_{\lambda}^{i}(., y), D \boldsymbol{u}_{\lambda}^{i}(., y)\right)_{2} \leq(1 / 2)\left([\boldsymbol{a}+\boldsymbol{H}] \boldsymbol{\xi}_{i}(., y), \boldsymbol{\xi}_{i}(., y)\right)_{2}+\epsilon(\lambda)$,

where the function $\epsilon(\lambda)$ exactly matches $(1 / 2)\left([\boldsymbol{a}+\boldsymbol{H}] \boldsymbol{\xi}^{i}(., y),\left(D \boldsymbol{u}_{\lambda}^{i}-\boldsymbol{\xi}^{i}\right)(., y)\right)_{2}$ and so converges to 0 as $\lambda$ goes to 0 . Because of the antisymmetry of $\boldsymbol{H}(\cdot, y)$, we deduce

$$
\limsup _{\lambda \rightarrow 0}\left(\boldsymbol{a} D \boldsymbol{u}_{\lambda}^{i}(., y), D \boldsymbol{u}_{\lambda}^{i}(., y)\right)_{2} \leq\left(\boldsymbol{a} \boldsymbol{\xi}_{i}(., y), \boldsymbol{\xi}_{i}(., y)\right)_{2} .
$$

Since $(\boldsymbol{a}(., y) ., .)_{2}$ is equivalent on $\mathbb{D}$ to the canonical inner product (Assumption 2.3), we deduce that the convergence of $\left(D \boldsymbol{u}_{\lambda}^{i}(., y)\right)_{\lambda}$ to $\boldsymbol{\xi}_{i}$ holds in the strong sense in $\mathbb{D}$. In particular

$$
\lambda\left|\boldsymbol{u}_{\lambda}^{i}(., y)\right|_{2}^{2}+\left|D \boldsymbol{u}_{\lambda}^{i}(., y)-\boldsymbol{\xi}_{i}(., y)\right|_{2}^{2} \underset{\lambda \rightarrow 0}{\longrightarrow} 0 .
$$

This proves the first part of the statement for the function $\boldsymbol{u}_{\lambda}^{i}(., y)$. The second part results from Proposition 4.1 statement (21a) (with $C_{2}=0$ ). The same job can be carried out for the successive derivatives up to order 2 of $\boldsymbol{u}_{\lambda}^{i}(., y)$.

\section{The Itô Formula}

In this section, our objective is to apply the Itô formula to the process $\left(\bar{X}^{\varepsilon}, X^{\varepsilon}\right)$ and to the function $(x, y) \mapsto w_{\lambda}(\omega, x, y)$, where $\boldsymbol{w}_{\lambda}$ is defined as the solution of the equation

$$
\lambda \boldsymbol{w}_{\lambda}-\boldsymbol{L}^{y} \boldsymbol{w}_{\lambda}=\boldsymbol{h}(., y)+\boldsymbol{f}(., y) .
$$

The functions $\boldsymbol{h}(., y)$ and $\boldsymbol{f}(., y)$ satisfy the assumptions of Proposition 4.1. Even if the function $(x, y) \mapsto w_{\lambda}(\omega, x, y)$ is not of class $C^{2}$, we will prove that the regularity properties stated in this latter proposition are sufficient to apply the Itô formula by means of a smooth approximating sequence $\left(\boldsymbol{w}_{\lambda}^{m}\right)_{m}$ of the function $\boldsymbol{w}_{\lambda}$ and estimates on the transition densities of the process $X^{\varepsilon}$. This approximation has to converge towards $\boldsymbol{w}_{\lambda}$ as $m$ goes to $\infty$ in a certain sense. Actually the main difficulty lies in the convergence of the second order derivatives with respect to the random medium. That is essentially this point we are going to discuss.

For any $\boldsymbol{\varphi} \in L^{2}(\Omega), k=1, \ldots, d$ and $r \in \mathbb{R}^{*}$, we define $\Gamma_{r}^{k} \boldsymbol{\varphi}=\frac{1}{r}\left(T_{r e_{k}} \boldsymbol{\varphi}-\boldsymbol{\varphi}\right)$. It is plain to check that this operator satisfies the following properties:

$$
\begin{gathered}
\boldsymbol{\varphi}, \boldsymbol{\psi} \in L^{2}(\Omega), \quad\left(\Gamma_{r}^{k} \boldsymbol{\varphi}, \boldsymbol{\psi}\right)_{2}=-\left(\boldsymbol{\varphi}, \Gamma_{-r}^{k} \boldsymbol{\psi}\right)_{2}, \\
\forall \boldsymbol{\varphi} \in \mathbb{H}_{1}, \quad\left|\Gamma_{r}^{k} \boldsymbol{\varphi}\right|_{2} \leq\|\boldsymbol{\varphi}\|_{1} .
\end{gathered}
$$

We now adapt the ideas of Gilbarg Trudinger [6, Sect. 8.3] to this framework. 
Proposition 5.1. For each $y \in \mathbb{R}^{d}, D^{2} \boldsymbol{w}_{\lambda}$ is a stationary random fields and $\left|D^{2} \boldsymbol{w}_{\lambda}(., y)\right|_{2}$ is bounded independently of $y \in \mathbb{R}^{d}$. Furthermore, for any $y, h \in \mathbb{R}^{d}$

$$
\left|D^{2} \boldsymbol{w}_{\lambda}(., y+h)-D^{2} \boldsymbol{w}_{\lambda}(., y)\right|_{2} \leq C|h|,
$$

where the constant $C$ does not depend on $y, h \in \mathbb{R}^{d}$.

Proof: Remind that we have: $\forall \boldsymbol{\varphi} \in \mathcal{C}, \lambda \boldsymbol{w}_{\lambda}-\boldsymbol{L}^{y} \boldsymbol{w}_{\lambda}=\boldsymbol{h}+\boldsymbol{f}$. Note that the parameter $y \in \mathbb{R}^{d}$ is temporarily omitted to simplify the notations. Let us consider $r \in \mathbb{R}^{*}$ and $k \in\{1, \ldots, d\}$. For any $\varphi \in \mathcal{C}$, we obtain:

$$
\begin{aligned}
\left([\boldsymbol{h}+\boldsymbol{f}], \Gamma_{r}^{k} \boldsymbol{\varphi}\right)_{2}= & \lambda\left(\boldsymbol{w}_{\lambda}, \Gamma_{r}^{k} \boldsymbol{\varphi}\right)_{2}+(1 / 2)\left([\boldsymbol{a}+\boldsymbol{H}] D \boldsymbol{w}_{\lambda}, D \Gamma_{r}^{k} \boldsymbol{\varphi}\right)_{2} \\
= & \lambda\left(\boldsymbol{w}_{\lambda}, \Gamma_{r}^{k} \boldsymbol{\varphi}\right)_{2}-(1 / 2)\left([\boldsymbol{a}+\boldsymbol{H}] D \Gamma_{-r}^{k} \boldsymbol{w}_{\lambda}, D \boldsymbol{\varphi}\right)_{2} \\
& -(1 / 2)\left(\left[\Gamma_{-r}^{k} \boldsymbol{a}+\Gamma_{-r}^{k} \boldsymbol{H}\right] D \boldsymbol{w}_{\lambda}, D \boldsymbol{\varphi}\right)_{2},
\end{aligned}
$$

so that we deduce

$$
(1 / 2)\left([\boldsymbol{a}+\boldsymbol{H}] D \Gamma_{-r}^{k} \boldsymbol{w}_{\lambda}, D \boldsymbol{\varphi}\right)_{2} \leq\left(|\boldsymbol{h}+\boldsymbol{f}|_{2}+\left|\lambda \boldsymbol{w}_{\lambda}\right|_{2}+\Lambda\left\|\boldsymbol{w}_{\lambda}\right\|_{1}\right)\|\boldsymbol{\varphi}\|_{1} .
$$

From (21a), $\sup _{r \in \mathbb{R}^{*}}\left|D \Gamma_{r}^{k} \boldsymbol{w}_{\lambda}(., y)\right|_{2}$ is bounded (independently of $y \in \mathbb{R}^{d}$ ). For each $y \in \mathbb{R}^{d}$, up to extracting a subsequence, the family $\left(\Gamma_{r}^{k} \boldsymbol{w}_{\lambda}(., y)\right)_{r}$ admits a weak limit in $\mathbb{H}_{1}$ as $r \rightarrow 0$, denoted by $\boldsymbol{F}_{\lambda}^{k}(., y)$. As guessed by the reader, $\boldsymbol{F}_{\lambda}^{k}(., y)$ is actually a weak solution of the equation:

$\lambda\left(\boldsymbol{F}_{\lambda}^{k}, \boldsymbol{\varphi}\right)_{2}+(1 / 2)\left([\boldsymbol{a}+\boldsymbol{H}] D \boldsymbol{F}_{\lambda}^{k}, D \boldsymbol{\varphi}\right)_{2}=-\left(\boldsymbol{h}+\boldsymbol{f}, D_{k} \boldsymbol{\varphi}\right)_{2}+(1 / 2)\left(\left[D_{k} \boldsymbol{a}+D_{k} \boldsymbol{H}\right] D \boldsymbol{w}_{\lambda}, D \boldsymbol{\varphi}\right)_{2}$.

This proves the uniqueness of the weak limit. The weak convergence of $\left(\Gamma_{r}^{k} \boldsymbol{w}_{\lambda}\right)_{r}$ in $\mathbb{H}_{1}$ thus holds as $r \rightarrow 0$ (and not up to a subsequence). In particular, it results that $\left(D_{j} \boldsymbol{F}_{\lambda}^{k}(., y), \boldsymbol{\varphi}\right)_{2}=$ $-\left(D_{k} \boldsymbol{w}_{\lambda}, D_{j} \boldsymbol{\varphi}\right)_{2}$ for any $\boldsymbol{\varphi} \in \operatorname{Dom}\left(D_{j}\right)$ and $1 \leq j, k \leq d$. Since the operator $D_{j}$ is antisymmetric and closed, this also proves that $D \boldsymbol{w}_{\lambda}(., y) \in \operatorname{Dom}(D)$ and $D^{2} \boldsymbol{w}_{\lambda}(., y)$ is a stationary random field.

The second part of the statement is quite similar to the corresponding part of the proof of Proposition 4.1 so that details are left to the reader.

Let us now consider a regularizing sequence of mollifiers $\left(\varrho_{m}\right)_{m} \in C_{c}^{\infty}\left(\mathbb{R}^{d} \times \mathbb{R}^{d}\right)$ (smooth functions with compact support) and define for any $m \in \mathbb{N}^{*},(\omega, y) \in \Omega \times \mathbb{R}^{d}$,

$$
\boldsymbol{w}_{\lambda}^{m}(\omega, y)=\int_{\mathbb{R}^{d} \times \mathbb{R}^{d}} \boldsymbol{w}_{\lambda}\left(\tau_{x^{\prime}} \omega, y-y^{\prime}\right) \varrho_{m}\left(x^{\prime}, y^{\prime}\right) d x^{\prime} d y^{\prime}
$$


Of course, the Itô formula holds for $\boldsymbol{w}_{\lambda}^{m}$, which is a smooth function:

$$
\begin{aligned}
\varepsilon d w_{\lambda}^{m}\left(\bar{X}_{t}^{\varepsilon}, X_{t}^{\varepsilon}\right)= & \varepsilon^{-1} L^{X_{t}^{\varepsilon}} w_{\lambda}^{m}\left(\bar{X}_{t}^{\varepsilon}, X_{t}^{\varepsilon}\right) d t+c \cdot D w_{\lambda}^{m}\left(\bar{X}_{t}^{\varepsilon}, X_{t}^{\varepsilon}\right) d t \\
& +D w_{\lambda}^{m} \cdot \sigma\left(\bar{X}_{t}^{\varepsilon}, X_{t}^{\varepsilon}\right) d B_{t}+b \partial_{y} w_{\lambda}^{m}\left(\bar{X}_{t}^{\varepsilon}, X_{t}^{\varepsilon}\right) d t \\
& +\varepsilon\left(\partial_{y} w_{\lambda}^{m}\right)^{*} \sigma\left(\bar{X}_{t}^{\varepsilon}, X_{t}^{\varepsilon}\right) d B_{t}+\varepsilon c \cdot \partial_{y} w_{\lambda}^{m}\left(\bar{X}_{t}^{\varepsilon}, X_{t}^{\varepsilon}\right) d t \\
& +(\varepsilon / 2) \operatorname{trace}\left(a \partial_{y y}^{2} w_{\lambda}^{m}\right)\left(\bar{X}_{t}^{\varepsilon}, X_{t}^{\varepsilon}\right) d t+\operatorname{trace}\left(a D \partial_{y} w_{\lambda}^{m}\right)\left(\bar{X}_{t}^{\varepsilon}, X_{t}^{\varepsilon}\right) d t .
\end{aligned}
$$

It remains to let $m$ go to $\infty$. From the regularity properties of $\boldsymbol{w}_{\lambda}$ stated in Propositions 5.1 and 4.1, it is plain to check that the derivatives of $\boldsymbol{w}_{\lambda}^{m}$ involved in (30) can be expressed as follows: the functions $\boldsymbol{g}_{\lambda}^{m} \equiv \partial_{y_{j}} \boldsymbol{w}_{\lambda}^{m}$ (resp. $\partial_{y_{j} y_{k}}^{2} \boldsymbol{w}_{\lambda}^{m}$, resp. $D_{j} \boldsymbol{w}_{\lambda}^{m}$, resp. $D_{j k}^{2} \boldsymbol{w}_{\lambda}^{m}$, resp. $D_{k} \partial_{y_{j}} \boldsymbol{w}_{\lambda}^{m}$ ) and $\boldsymbol{g}_{\lambda} \equiv \partial_{y_{j}} \boldsymbol{w}_{\lambda}$ (resp. $\partial_{y_{j} y_{k}}^{2} \boldsymbol{w}_{\lambda}$, resp. $D_{j} \boldsymbol{w}_{\lambda}$, resp. $D_{j k}^{2} \boldsymbol{w}_{\lambda}$, resp. $D_{k} \partial_{y_{j}} \boldsymbol{w}_{\lambda}$ ) satisfy

$$
\boldsymbol{g}_{\lambda}^{m}(\omega, y)=\int_{\mathbb{R}^{d} \times \mathbb{R}^{d}} \boldsymbol{g}_{\lambda}\left(\tau_{x^{\prime}} \omega, y-y^{\prime}\right) \varrho_{m}\left(x^{\prime}, y^{\prime}\right) d x^{\prime} d y^{\prime}
$$

The convergence of the derivatives of $\boldsymbol{w}_{\lambda}^{m}$ towards the corresponding derivatives of $\boldsymbol{w}_{\lambda}$ (Lemma 5.3 below) and estimates on the process $X^{\varepsilon}$ (Lemma 5.2 below) enable to pass to the limit, a $m$ goes to $\infty$, in (30).

Lemma 5.2. There exists a positive constant $A$, only depending on $M, \Lambda, d$, such that the function $f_{5.2}: \mathbb{R} \times \mathbb{R}^{d} \times \mathbb{R}^{d} \rightarrow \mathbb{R}$ defined by $f_{5.2}(t, x, y)=\frac{A}{t^{d / 2}} e^{-\frac{|y-x|^{2}}{A t}}$ satisfies, for any measurable positive function $\varphi$ defined on $\Omega \times \mathbb{R}^{d}$ and $t>0$,

$$
\overline{\mathbb{E}}_{x}^{\varepsilon}\left[\varphi\left(\bar{X}_{t}^{\varepsilon}, X_{t}^{\varepsilon}\right)\right] \leq \mathbb{M} \int_{\mathbb{R}^{d}} \varphi(\omega, y) f_{5.2}(t, x, y) d y
$$

Notations. To relieve the notations, for each $t>0$ and $x \in \mathbb{R}^{d}$, the expectations with respect to the probability measures $f_{5.2}(t, x, y) d \mu \otimes d y$ and $\left(\int_{0}^{t} f_{5.2}(r, x, y) d r\right) d \mu \otimes d y$ on $\Omega \times \mathbb{R}^{d}$ are respectively denoted by $\mathbb{M}_{5.2}^{t}$ and $\widetilde{\mathbb{M}}_{5.2}^{t}$. Note that the parameter $x$ is omitted from these notations.

Lemma 5.3. Suppose that a measurable function $\boldsymbol{w}: \Omega \times \mathbb{R}^{d} \rightarrow \mathbb{R}$ satisfies

$$
\forall(y, h) \in \mathbb{R}^{d} \times \mathbb{R}^{d}, \quad|\boldsymbol{w}(\cdot, y)|_{2} \leq C, \quad \text { and } \quad|\boldsymbol{w}(\cdot, y+h)-\boldsymbol{w}(\cdot, y)|_{2} \leq C|h|,
$$

and define $\boldsymbol{w}^{m}(\omega, y)=\int_{\mathbb{R}^{d} \times \mathbb{R}^{d}} \boldsymbol{w}\left(\tau_{x^{\prime}} \omega, y-y^{\prime}\right) \varrho_{m}\left(x^{\prime}, y^{\prime}\right) d x^{\prime} d y^{\prime}$. Then, for any $t>0$

$$
\overline{\mathbb{E}}_{x}^{\varepsilon}\left[\left|\boldsymbol{w}^{m}\left(\bar{X}_{t}^{\varepsilon}, X_{t}^{\varepsilon}\right)-\boldsymbol{w}\left(\bar{X}_{t}^{\varepsilon}, X_{t}^{\varepsilon}\right)\right|\right]+\overline{\mathbb{E}}_{x}^{\varepsilon}\left[\int_{0}^{t}\left|\boldsymbol{w}^{m}\left(\bar{X}_{s}^{\varepsilon}, X_{s}^{\varepsilon}\right)-\boldsymbol{w}\left(\bar{X}_{s}^{\varepsilon}, X_{s}^{\varepsilon}\right)\right| d s\right] \stackrel{m \rightarrow \infty}{\longrightarrow} 0 .
$$


Proof of Lemma 5.2: It easily results from the Aronson estimates (cf. [13]): there exists a constant $A$ that only depends on $M, \Lambda, d$ such that $\forall \varepsilon>0, \forall \omega \in \Omega, \forall(t, x, y) \in \mathbb{R}_{+}^{*} \times \mathbb{R}^{d} \times \mathbb{R}^{d}$

$$
\frac{1}{A t^{d / 2}} e^{-\frac{A|y-x|^{2}}{t}} \leq p^{\varepsilon, \omega}(0, x, t, y) \leq \frac{A}{t^{d / 2}} e^{-\frac{|y-x|^{2}}{A t}},
$$

where $p^{\varepsilon, \omega}$ denote the transition densities of the Markov process $X^{\varepsilon}$.

Proof of Lemma 5.3: From Lemma 5.2, we have

$$
\begin{aligned}
& \overline{\mathbb{E}}_{x}^{\varepsilon}\left[\left|\boldsymbol{w}^{m}\left(\bar{X}_{t}^{\varepsilon}, X_{t}^{\varepsilon}\right)-\boldsymbol{w}\left(\bar{X}_{t}^{\varepsilon}, X_{t}^{\varepsilon}\right)\right|\right] \\
& \leq \mathbb{M}_{5.2}^{t}\left[\left|\boldsymbol{w}^{m}-\boldsymbol{w}\right|\right] \\
& \leq \int_{\left(\mathbb{R}^{d}\right)^{3}}\left|\boldsymbol{w}\left(\tau_{x^{\prime}} \omega, y-y^{\prime}\right)-\boldsymbol{w}(\omega, y)\right|_{2} f_{5.2}(t, x, y) \varrho_{m}\left(x^{\prime}, y^{\prime}\right) d y d x^{\prime} d y^{\prime} \\
& \leq \int_{\left(\mathbb{R}^{d}\right)^{3}}\left|\boldsymbol{w}\left(\tau_{x^{\prime}} \omega, y-y^{\prime}\right)-\boldsymbol{w}\left(\omega, y-y^{\prime}\right)\right|_{2} f_{5.2}(t, x, y) \varrho_{m}\left(x^{\prime}, y^{\prime}\right) d y d x^{\prime} d y^{\prime} \\
&+\int_{\left(\mathbb{R}^{d}\right)^{3}}\left|\boldsymbol{w}\left(\omega, y-y^{\prime}\right)-\boldsymbol{w}(\omega, y)\right|_{2} f_{5.2}(t, x, y) \varrho_{m}\left(x^{\prime}, y^{\prime}\right) d y d x^{\prime} d y^{\prime} .
\end{aligned}
$$

Because of the stochastic continuity of the translation operators on the medium, the term $\left|\boldsymbol{w}\left(\tau_{x^{\prime}} \omega, y-y^{\prime}\right)-\boldsymbol{w}\left(\omega, y-y^{\prime}\right)\right|_{2}$ tends to 0 as $x^{\prime}$ goes to 0 (and is bounded independently of $y-y^{\prime} \in \mathbb{R}^{d}$ ) so that classical convolution technics ensure that the first term in the above right-hand side converges to 0 as $m$ goes to $\infty$. Concerning the second term, it also converges to 0 , as $m$ goes to $\infty$, because of the estimate $\left|\boldsymbol{w}\left(\omega, y-y^{\prime}\right)-\boldsymbol{w}(\omega, y)\right|_{2} \leq \min \left(2 C,\left|y^{\prime}\right|\right)$ and classical convolution technics again.

The second term in the left-hand side of (31) raises no additional difficulty. So details are left to the reader.

We sum up this discussion in the following

Theorem 5.4. Let $\boldsymbol{h}, \boldsymbol{f}$ be two functions satisfying the assumptions of Proposition 4.1. Let $\boldsymbol{w}_{\lambda}$ be the solution of the resolvent equation:

$$
\lambda \boldsymbol{w}_{\lambda}(\cdot, y)-\boldsymbol{L}^{y} \boldsymbol{w}_{\lambda}(\cdot, y)=\boldsymbol{h}(\cdot, y)+\boldsymbol{f}(\cdot, y) .
$$

Then the following Itô formula holds:

$$
\begin{aligned}
\varepsilon d w_{\lambda}\left(\bar{X}_{t}^{\varepsilon}, X_{t}^{\varepsilon}\right)= & \varepsilon^{-1}\left(\lambda w_{\lambda}-h-f\right)\left(\bar{X}_{t}^{\varepsilon}, X_{t}^{\varepsilon}\right) d t+c \cdot D w_{\lambda}\left(\bar{X}_{t}^{\varepsilon}, X_{t}^{\varepsilon}\right) d t \\
& +D w_{\lambda} \cdot \sigma\left(\bar{X}_{t}^{\varepsilon}, X_{t}^{\varepsilon}\right) d B_{t}+b \partial_{y} w_{\lambda}\left(\bar{X}_{t}^{\varepsilon}, X_{t}^{\varepsilon}\right) d t \\
& +\varepsilon\left(\partial_{y} w_{\lambda}\right)^{*} \sigma\left(\bar{X}_{t}^{\varepsilon}, X_{t}^{\varepsilon}\right) d B_{t}+\varepsilon c \cdot \partial_{y} w_{\lambda}\left(\bar{X}_{t}^{\varepsilon}, X_{t}^{\varepsilon}\right) d t \\
& +(\varepsilon / 2) \operatorname{trace}\left(a \partial_{y y}^{2} w_{\lambda}\right)\left(\bar{X}_{t}^{\varepsilon}, X_{t}^{\varepsilon}\right) d t+\operatorname{trace}\left(a D \partial_{y} w_{\lambda}\right)\left(\bar{X}_{t}^{\varepsilon}, X_{t}^{\varepsilon}\right) d t
\end{aligned}
$$




\section{Asymptotic Theorems}

Classical ergodic theorem. Let us now investigate the asymptotic behavior, as $\varepsilon \rightarrow 0$, of functionals of the type $\int_{0}^{t} \Psi\left(\bar{X}_{r}^{\varepsilon}, X_{r}^{\varepsilon}\right) d r$ for a suitable locally stationary random field $\Psi$. This behavior is very intuitive: this functional averages with respect to its first variable, that is at a small scale, while the second variable prescribes the behavior at a large scale. More precisely

\section{Theorem 6.1. (Ergodic Theorem)}

Let us consider $\Psi: \Omega \times \mathbb{R}^{d} \rightarrow \mathbb{R}$ such that $\widetilde{\mathbb{M}}_{5.2}^{t}[|\Psi|]<+\infty$. Denoting $\bar{\Psi}(y)=\mathbb{M}[\Psi(\cdot, y)]$, the following convergence holds:

$$
\overline{\mathbb{E}_{x}^{\varepsilon}}\left[\sup _{0 \leq s \leq t}\left|\int_{0}^{s} \Psi\left(\bar{X}_{r}^{\varepsilon}, X_{r}^{\varepsilon}\right)-\bar{\Psi}\left(X_{r}^{\varepsilon}\right) d r\right|\right] \underset{\varepsilon \rightarrow 0}{\longrightarrow} 0
$$

Proof: The proof of this theorem is split up into two steps. The first one consists in proving this result for a bounded smooth function $\Psi$. The second step extends the result to the general case.

First step: We consider a bounded function $\boldsymbol{\Psi}(\omega, y)=\boldsymbol{\varphi}(\omega) g(y)$, with $\boldsymbol{\varphi} \in \mathcal{C}, g(y) \in$ $C_{b}^{3}\left(\mathbb{R}^{d}\right)$ (bounded, three times continuously differentiable with bounded derivatives up to order three). Obviously, Theorem 5.4 applies and provides us with the Itô formula:

$$
\begin{aligned}
d v_{\lambda}\left(\bar{X}_{t}^{\varepsilon}, X_{t}^{\varepsilon}\right)= & -\varepsilon^{-2}\left(\Psi\left(\bar{X}_{t}^{\varepsilon}, X_{t}^{\varepsilon}\right)-\lambda v_{\lambda}\left(\bar{X}_{t}^{\varepsilon}, X_{t}^{\varepsilon}\right)\right) d t \\
& +\varepsilon^{-1} c \cdot D v_{\lambda}\left(\bar{X}_{t}^{\varepsilon}, X_{t}^{\varepsilon}\right) d t+\varepsilon^{-1} D v_{\lambda} \cdot \sigma\left(\bar{X}_{t}^{\varepsilon}, X_{t}^{\varepsilon}\right) d B_{t} \\
& +\left(\partial_{y} v_{\lambda}\right)^{*} \sigma\left(\bar{X}_{t}^{\varepsilon}, X_{t}^{\varepsilon}\right) d B_{t}+\varepsilon c \cdot \partial_{y} v_{\lambda}\left(\bar{X}_{t}^{\varepsilon}, X_{t}^{\varepsilon}\right) d t \\
& +(1 / 2) \operatorname{trace}\left[a \partial_{y y}^{2} v_{\lambda}\right]\left(\bar{X}_{t}^{\varepsilon}, X_{t}^{\varepsilon}\right) d t+\varepsilon^{-1} b \partial_{y} v_{\lambda}\left(\bar{X}_{t}^{\varepsilon}, X_{t}^{\varepsilon}\right) d t \\
& +\varepsilon^{-1} \operatorname{trace}\left[a D \partial_{y} v_{\lambda}\right]\left(\bar{X}_{t}^{\varepsilon}, X_{t}^{\varepsilon}\right) d t .
\end{aligned}
$$

We deduce (the constant $C$ may change from line to line)

$$
\begin{aligned}
\overline{\mathbb{E}}_{x}^{\varepsilon}\left[\sup _{0 \leq s \leq t}\left|\int_{0}^{s}\left[\Psi-\lambda v_{\lambda}\right]\left(\bar{X}_{r}^{\varepsilon}, X_{r}^{\varepsilon}\right) d r\right|^{2}\right] \leq & C \varepsilon^{2} \overline{\mathbb{E}}_{x}^{\varepsilon}\left[\sup _{0 \leq s \leq t}\left|v_{\lambda}\left(\bar{X}_{s}^{\varepsilon}, X_{s}^{\varepsilon}\right)\right|^{2}\right] \\
& +C t \overline{\mathbb{E}}_{x}^{\varepsilon}\left[\int_{0}^{t}\left|\varepsilon c \cdot D v_{\lambda}\left(\bar{X}_{r}^{\varepsilon}, X_{r}^{\varepsilon}\right)\right|^{2} d r\right] \\
& +\varepsilon^{2} \Delta_{1}^{\varepsilon, \lambda}+\varepsilon^{4} \Delta_{2}^{\varepsilon, \lambda} .
\end{aligned}
$$

Here $\Delta_{1}^{\varepsilon, \lambda}$ and $\Delta_{2}^{\varepsilon, \lambda}$ stand for the other terms that are not written but can be treated in the same way as the second one. Fix $\lambda>0$. From the boundedness of $\boldsymbol{\Psi}, \boldsymbol{v}_{\lambda}$ is bounded $\left(\left(G_{\lambda}^{y}\right)_{\lambda}\right.$ is a resolvent operator family associated to a $L^{\infty}(\Omega)$-contraction semigroup, see [11, Sect. 2.2]). 
So the first term of the right-hand side vanishes as $\varepsilon$ tends to 0 . We now turn to the second term

$$
\overline{\mathbb{E}}_{x}^{\varepsilon}\left[\int_{0}^{t}\left|\varepsilon c \cdot D v_{\lambda}\left(\bar{X}_{r}^{\varepsilon}, X_{r}^{\varepsilon}\right)\right|^{2} d r\right] \stackrel{\text { Lemma } 5.2}{\leq} \varepsilon^{2} \widetilde{\mathbb{M}}_{5.2}^{t}\left[\left|\boldsymbol{c} \cdot D \boldsymbol{v}_{\lambda}\right|^{2}\right],
$$

From the boundedness of $\boldsymbol{c}$ (see Assumption 2.2) and $\left|D \boldsymbol{v}_{\lambda}(., y)\right|_{2}$ (cf. (21a)), this latter quantity tends to 0 as $\varepsilon$ goes to 0 .

From Lemma 6.2 below and (21a), which implies that $\sup _{y \in \mathbb{R}^{d}}\left|\lambda \boldsymbol{v}_{\lambda}(\cdot, y)-\bar{\Psi}(y)\right|_{2}<+\infty$, we obtain:

$$
\begin{gathered}
\overline{\mathbb{E}}_{x}^{\varepsilon}\left[\sup _{0 \leq s \leq t}\left|\int_{0}^{s}\left[\lambda v_{\lambda}-\bar{\Psi}\right]\left(\bar{X}_{r}^{\varepsilon}, X_{r}^{\varepsilon}\right) d r\right|^{2}\right] \leq t \widetilde{\mathbb{M}}_{5.2}^{t}\left[\left|\lambda \boldsymbol{v}_{\lambda}-\bar{\Psi}\right|^{2}\right] \\
\stackrel{\text { Lemma }}{=}{ }^{6.2} \delta(\lambda)
\end{gathered}
$$

where $\lim _{\lambda \rightarrow 0} \delta(\lambda)=0$. The result follows in this case by fixing first $\lambda$ small enough to make $\delta(\lambda)$ small and then choosing $\varepsilon$ small enough to make the right-hand side of (34) small too.

Second step: Let us now generalize the class of considered functions $\Psi$. Let us only assume that $\widetilde{\mathbb{M}}_{5.2}^{t}[|\Psi|]<+\infty$. Thanks to density arguments, we can find $\left(\boldsymbol{\varphi}_{n}\right)_{n} \in \mathcal{C}^{\mathbb{N}}$ and $\left(g_{n}\right)_{n} \in C_{b}^{3}\left(\mathbb{R}^{d}\right)^{\mathbb{N}}$ such that $\widetilde{\mathbb{M}}_{5.2}^{t}\left[\left|\Psi-\boldsymbol{\varphi}_{n} g_{n}\right|^{2}\right] \rightarrow 0$ as $n$ tends to 0 . As guessed by the reader, we obviously conclude with the help of Lemma 5.2.

Lemma 6.2. For each fixed $y \in \mathbb{R}^{d}$, the solution $\boldsymbol{v}_{\lambda}(., y)$ of the equation $\left(\lambda-\boldsymbol{L}^{y}\right) \boldsymbol{v}_{\lambda}(., y)=$ $\boldsymbol{\Psi}(., y)$ satisfies $\left|\lambda \boldsymbol{v}_{\lambda}(., y)-\bar{\Psi}(., y)\right|_{2} \rightarrow 0$ as $\lambda$ goes to 0 .

Proof: Apply Proposition 4.1 with the functions $\boldsymbol{h}=\boldsymbol{\Psi}$ and $\boldsymbol{f}=0$. From (21a), the family $\left(\lambda \boldsymbol{v}_{\lambda}(., y)\right)_{\lambda}$ is bounded in $L^{2}(\Omega)$. So we can extract a $L^{2}(\Omega)$-weakly converging subsequence, still indexed with $\lambda>0$, and let $\boldsymbol{g} \in L^{2}(\Omega)$ denote its limit.

The weak form of the resolvent equation reads, for $\varphi \in \mathbb{H}_{1}$,

$$
\lambda\left(\boldsymbol{v}_{\lambda}(., y), \boldsymbol{\varphi}\right)_{2}+(1 / 2)\left([\boldsymbol{a}+\boldsymbol{H}] D \boldsymbol{v}_{\lambda}(., y), D \boldsymbol{\varphi}\right)_{2}=(\boldsymbol{\Psi}(., y), \boldsymbol{\varphi})_{2} .
$$

Remind that $\boldsymbol{L}^{y *}$ stands for the adjoint operator of $\boldsymbol{L}^{y}$ in $L^{2}(\Omega, \mu)$. If $\boldsymbol{\varphi} \in \operatorname{Dom}\left(\left(\boldsymbol{L}^{y}\right)^{*}\right)$, (35) also reads $\lambda\left(\boldsymbol{v}_{\lambda}(., y), \boldsymbol{\varphi}\right)_{2}+\left(\boldsymbol{v}_{\lambda}(., y),\left(\boldsymbol{L}^{y}\right)^{*} \boldsymbol{\varphi}\right)_{2}=(\boldsymbol{\Psi}, \boldsymbol{\varphi})_{2}$. Multiplying this equality by $\lambda$ and passing to the limit as $\lambda$ goes to 0 leads to $\left(\boldsymbol{g},\left(\boldsymbol{L}^{y}\right)^{*} \boldsymbol{\varphi}\right)_{2}=0$ for any $\boldsymbol{\varphi} \in \operatorname{Dom}\left(\left(\boldsymbol{L}^{y}\right)^{*}\right)$. In particular (see Section 4), $\boldsymbol{g} \in \operatorname{Dom}\left(\boldsymbol{L}^{y}\right) \subset \mathbb{H}_{1}$ and $\boldsymbol{L}^{y} \boldsymbol{g}=0$. From Assumption 2.3, $\|\boldsymbol{g}\|_{1}^{2} \leq M\|\boldsymbol{g}\|_{1, y}^{2}=-\left(\boldsymbol{g}, \boldsymbol{L}^{y} \boldsymbol{g}\right)_{2}=0$. So we get $D_{i} \boldsymbol{g}=0, \forall i=1, \ldots, d$. As a consequence, $\boldsymbol{g}$ is invariant under space translations. From Definition $2.1, \boldsymbol{g}$ must be constant $\mu$ almost surely and then necessarily equal to

$$
\boldsymbol{g}=\lim _{\lambda \rightarrow 0} \mathbb{M}\left[\lambda \boldsymbol{v}_{\lambda}(., y)\right]=\lim _{\lambda \rightarrow 0} \mathbb{M}\left[\lambda \boldsymbol{v}_{\lambda}(., y)-\boldsymbol{L}^{y} \boldsymbol{v}_{\lambda}(., y)\right]=\lim _{\lambda \rightarrow 0} \mathbb{M}[\mathbf{\Psi}(., y)]
$$


This proves the uniqueness of the weak limit.

Choosing $\varphi=\lambda \boldsymbol{v}_{\lambda}$ in (35) leads to

$\lim _{\lambda \rightarrow 0}\left[\lambda^{2}\left|\boldsymbol{v}_{\lambda}(., y)\right|_{2}^{2}+(\lambda / 2)\left(\boldsymbol{a} D \boldsymbol{v}_{\lambda}(., y), D \boldsymbol{v}_{\lambda}(., y)\right)_{2}\right]=\lim _{\lambda \rightarrow 0}\left(\boldsymbol{\Psi}(., y), \lambda \boldsymbol{v}_{\lambda}(., y)\right)_{2}=\mathbb{M}[\boldsymbol{\Psi}(., y)]^{2}$

In particular, lim $\sup _{\lambda \rightarrow 0} \lambda^{2}\left|\boldsymbol{v}_{\lambda}(., y)\right|_{2}^{2} \leq|\boldsymbol{g}|_{2}^{2}$, from which the strong convergence in $L^{2}(\Omega)$ results.

Asymptotic theorem for highly oscillating functionals. Theorem 6.1 settles the issue of the asymptotic behavior of functionals of the form $\int_{0}^{t} \Psi\left(\bar{X}_{r}^{\varepsilon}, X_{r}^{\varepsilon}\right) d r$ for a locally random field $\Psi \in L^{1}\left(\Omega \times \mathbb{R}^{d} ; \widetilde{\mathbb{M}}_{5.2}^{t}\right)$. As explained in Section 1 and unlike the locally periodic case (see [2] or [1]), the locally stationary framework raises the issue of describing the asymptotic behavior of functionals of the type $\int_{0}^{t} \Psi_{\varepsilon}\left(\bar{X}_{r}^{\varepsilon}, X_{r}^{\varepsilon}\right) d r$ for a family $\left(\Psi_{\varepsilon}\right)_{\varepsilon}$ that is not convergent in $L^{1}\left(\Omega \times \mathbb{R}^{d} ; \widetilde{\mathbb{M}}_{5.2}^{t}\right)$. Theorem 6.3 states that these functionals converge to 0 as $\varepsilon$ goes to 0 provided that we can suitably control a sort of Poincaré inequality for the family $\left(\Psi_{\varepsilon}\right)_{\varepsilon}$. More precisely

Theorem 6.3. (Ergodic theorem II) For each $\varepsilon>0$, let us consider a function $\boldsymbol{\Psi}_{\varepsilon}$ such that $\int_{\mathbb{R}^{d}}\left|\Psi_{\varepsilon}(\cdot, y)\right|_{2}^{2} d y<+\infty$. Let us additionally assume that, for each $\varepsilon>0$, we can find a positive constant $C_{\varepsilon}$ such that the following type of "Poincaré inequality" holds: for any $\boldsymbol{\varphi}(\omega, y)=\chi(\omega) \varrho(y),(\chi, \varrho) \in \mathcal{C} \times C_{c}^{\infty}\left(\mathbb{R}^{d}\right)$,

$$
\mathbb{M}\left[\int_{\mathbb{R}^{d}} \boldsymbol{\Psi}_{\varepsilon} \boldsymbol{\varphi}(., y) d y\right] \leq C_{\varepsilon}\left(\mathbb{M}\left[\int_{\mathbb{R}^{d}}\left|\left(D+\varepsilon \partial_{y}\right) \boldsymbol{\varphi}(., y)\right|^{2} d y\right]\right)^{1 / 2},
$$

If $\varepsilon C_{\varepsilon} \rightarrow 0$ as $\varepsilon \rightarrow 0$ then $\forall t>0$,

$$
\overline{\mathbb{E}}_{x}^{\varepsilon}\left[\left|\int_{0}^{t} \Psi_{\varepsilon}\left(\bar{X}_{r}^{\varepsilon}, X_{r}^{\varepsilon}\right) d r\right|^{2}\right] \rightarrow 0, \quad \text { as } \varepsilon \rightarrow 0 .
$$

Proof: The proof of this result is divided in three steps:

First step (setup). Equip the set $\Omega \times \mathbb{R}^{d}$ with the product measure

$$
d \Pi(\omega, y)=(d \mu \otimes d y)(\omega, y) .
$$

Denote $(\cdot, \cdot)_{2, \Pi}$ the canonical inner product associated to the space $L^{2}\left(\Omega \times \mathbb{R}^{d}, \Pi\right),|\cdot|_{2, \Pi}$ the corresponding norm and $\mathcal{C}_{\Pi}$ the following subspace of $L^{2}\left(\Omega \times \mathbb{R}^{d}, \Pi\right)$ of smooth functions:

$$
\mathcal{C}_{\Pi}=\left\{\chi(\omega) \varrho(x) ;(\chi, \varrho) \in \mathcal{C} \times C_{c}^{\infty}\left(\mathbb{R}^{d}\right)\right\} .
$$


Let us now define the following unbounded operators on $\mathcal{C}_{\Pi} \subset L^{2}\left(\Omega \times \mathbb{R}^{d}, \Pi\right)$ :

$$
\forall \boldsymbol{\varphi} \in \mathcal{C}_{\Pi}, \quad \boldsymbol{L}^{\varepsilon} \boldsymbol{\varphi}=\frac{1}{2}\left(\operatorname{Div}+\varepsilon \operatorname{div}_{y}\right)\left[(\boldsymbol{a}+\boldsymbol{H})\left(\boldsymbol{D}+\varepsilon \partial_{y}\right) \boldsymbol{\varphi}\right]
$$

We now proceed as in [5, Ch. 3, Sect. 3] or [10, Ch. 1, Sect. 2] to extend this operator. We define, for any $\varphi, \psi \in \mathcal{C}_{\Pi}$,

$$
(\boldsymbol{\varphi}, \boldsymbol{\psi})_{1, \varepsilon}=\frac{1}{2} \int_{\Omega \times \mathbb{R}^{d}} \boldsymbol{a}\left[\left(\boldsymbol{D}+\varepsilon \partial_{y}\right) \boldsymbol{\psi}\right] \cdot\left[\left(D+\varepsilon \partial_{y}\right) \boldsymbol{\varphi}\right](\omega, y) d \Pi(\omega, y),
$$

and $\Theta_{\varepsilon}(\boldsymbol{\varphi}, \boldsymbol{\psi})=(\boldsymbol{\varphi}, \boldsymbol{\psi})_{2, \Pi}+(\boldsymbol{\varphi}, \boldsymbol{\psi})_{1, \varepsilon}$. For each $\varepsilon>0$, we can define $\mathbb{H}_{\Pi}^{\varepsilon}$ as the closure of $\mathcal{C}_{\Pi}$ in $L^{2}\left(\Omega \times \mathbb{R}^{d}, \Pi\right)$ with respect to the norm $\|\cdot\|_{1, \varepsilon}$ associated to the inner product $\Theta_{\varepsilon}$. Although the gradients $D \varphi$ and $\partial_{y} \varphi$ need not separately exist for $\varphi \in \mathbb{H}_{\Pi}^{\varepsilon}$, it makes sense to consider the "2-scale gradient" $\left(D+\varepsilon \partial_{y}\right) \varphi$ thanks to (38) and Assumption 2.3.

We now extend the operator $\boldsymbol{L}^{\varepsilon}$ as follows. For any $\alpha>0$, we define the following bilinear form on $\mathbb{H}_{\Pi}^{\varepsilon} \times \mathbb{H}_{\Pi}^{\varepsilon}$ :

$$
\mathcal{B}_{L}^{\alpha, \varepsilon}(\boldsymbol{\varphi}, \boldsymbol{\psi})=\alpha(\boldsymbol{\varphi}, \boldsymbol{\psi})_{2, \Pi}+\frac{1}{2} \int_{\Omega \times \mathbb{R}^{d}}(\boldsymbol{a}+\boldsymbol{H})\left[\left(\boldsymbol{D}+\varepsilon \partial_{y}\right) \boldsymbol{\psi}\right] \cdot\left[\left(D+\varepsilon \partial_{y}\right) \boldsymbol{\varphi}\right](\omega, y) d \Pi(\omega, y)
$$

This form is clearly continuous and coercive on $\mathbb{H}_{\Pi}^{\varepsilon} \times \mathbb{H}_{\Pi}^{\varepsilon}$. If, for some function $\varphi \in \mathbb{H}_{\Pi}^{\varepsilon}$, the application $\boldsymbol{\psi} \in \mathbb{H}_{\Pi}^{\varepsilon} \mapsto \mathcal{B}_{L}^{\alpha, \varepsilon}(\boldsymbol{\varphi}, \boldsymbol{\psi})$ is $L^{2}\left(\Omega \times \mathbb{R}^{d}, \Pi\right)$ continuous, then $\boldsymbol{\varphi} \in \operatorname{Dom}\left(\boldsymbol{L}^{\varepsilon}\right)$.

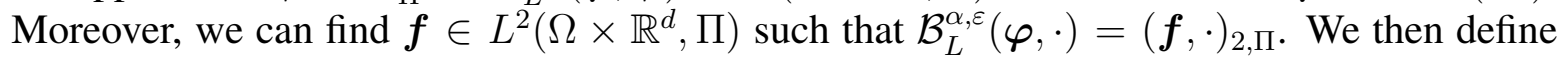
$\boldsymbol{L}^{\varepsilon} \boldsymbol{\varphi}=\alpha \boldsymbol{\varphi}-\boldsymbol{f}$. This definition does not depend on $\alpha$ and extends definition (37).

Second step (asymptotic control). We now aim at solving the resolvent equation, for each $\epsilon>0$

$$
\varepsilon^{2} \boldsymbol{\varphi}_{\varepsilon}-\boldsymbol{L}^{\varepsilon} \boldsymbol{\varphi}_{\varepsilon}=\boldsymbol{\Psi}_{\varepsilon}
$$

and at describing the asymptotic behavior, as $\varepsilon$ tends to 0 , of the solution $\varphi_{\varepsilon}$. From now on, $\mathcal{B}_{L}^{\varepsilon}$ denotes the bilinear form $\mathcal{B}_{L}^{\varepsilon^{2}, \varepsilon}$. Of course, from the coerciveness of $\mathcal{B}_{L}^{\varepsilon}$, we can find $\varphi_{\varepsilon} \in$ $\mathbb{H}_{\Pi}^{\varepsilon} \cap \operatorname{Dom}\left(\boldsymbol{L}^{\varepsilon}\right)$ that satisfies $\mathcal{B}_{L}^{\varepsilon}\left(\boldsymbol{\varphi}_{\varepsilon}, \boldsymbol{\varphi}\right)=\left(\boldsymbol{\Psi}_{\varepsilon}, \boldsymbol{\varphi}\right)_{2, \Pi}$ for any $\boldsymbol{\varphi} \in \mathbb{H}_{\Pi}^{\varepsilon}$, which is equivalent to (39). Choosing $\boldsymbol{\varphi}=\boldsymbol{\varphi}_{\varepsilon}$ and (36) leads to the estimate $\varepsilon^{2}\left|\boldsymbol{\varphi}_{\varepsilon}\right|_{2, \Pi}^{2}+(1 / 2)\left|\boldsymbol{\varphi}_{\varepsilon}\right|_{1, \varepsilon}^{2} \leq M C_{\varepsilon}^{2} / 2$. An easy consequence is $\varepsilon^{4}\left|\boldsymbol{\varphi}_{\varepsilon}\right|_{2, \Pi}^{2}+\left(\varepsilon^{2} / 2\right)\left\|\boldsymbol{\varphi}_{\varepsilon}\right\|_{1, \varepsilon}^{2} \leq \varepsilon^{2} M C_{\varepsilon}^{2} / 2 \rightarrow 0$ as $\varepsilon \rightarrow 0$.

Final step. From Lemma 6.4 below, the following formula holds $\overline{\mathbb{P}}_{x}^{\varepsilon}$ a.s.

$$
\begin{aligned}
d \varphi_{\varepsilon}\left(\bar{X}_{t}^{\varepsilon}, X_{t}^{\varepsilon}\right) & =\varepsilon^{-2} \boldsymbol{L}^{\varepsilon} \varphi_{\varepsilon}\left(\bar{X}_{t}^{\varepsilon}, X_{t}^{\varepsilon}\right) d t+\varepsilon^{-1} \sigma^{*}\left(D+\varepsilon \partial_{y}\right) \varphi_{\varepsilon}\left(\bar{X}_{t}^{\varepsilon}, X_{t}^{\varepsilon}\right) d B_{t} \\
& =\varepsilon^{-2}\left[\varepsilon^{2} \varphi_{\varepsilon}-\Psi_{\varepsilon}\right]\left(\bar{X}_{t}^{\varepsilon}, X_{t}^{\varepsilon}\right) d t+\varepsilon^{-1} \sigma^{*}\left(D+\varepsilon \partial_{y}\right) \varphi_{\varepsilon}\left(\bar{X}_{t}^{\varepsilon}, X_{t}^{\varepsilon}\right) d B_{t}
\end{aligned}
$$


and, as a consequence, for $0 \leq s \leq t$,

$$
\begin{aligned}
\int_{s}^{t} \Psi_{\varepsilon}\left(\bar{X}_{r}^{\varepsilon}, X_{r}^{\varepsilon}\right) d r= & -\varepsilon^{2}\left[\varphi_{\varepsilon}\left(\bar{X}_{t}^{\varepsilon}, X_{t}^{\varepsilon}\right)-\varphi_{\varepsilon}\left(\bar{X}_{s}^{\varepsilon}, X_{s}^{\varepsilon}\right)\right]+\varepsilon^{2} \int_{s}^{t} \varphi_{\varepsilon}\left(\bar{X}_{r}^{\varepsilon}, X_{r}^{\varepsilon}\right) d r \\
& +\varepsilon \int_{s}^{t} \sigma^{*}\left(D+\varepsilon \partial_{y}\right) \varphi_{\varepsilon}\left(\bar{X}_{r}^{\varepsilon}, X_{r}^{\varepsilon}\right) d B_{r}
\end{aligned}
$$

From Lemma 5.2, we deduce

$$
\overline{\mathbb{E}}_{x}^{\varepsilon}\left[\left|\int_{s}^{t} \Psi_{\varepsilon}\left(\bar{X}_{r}^{\varepsilon}, X_{r}^{\varepsilon}\right) d r\right|^{2}\right] \leq \varepsilon^{4} C(1+t)\left|\varphi_{\varepsilon}\right|_{2, \Pi}^{2}+\varepsilon^{2} C t\left\|\varphi_{\varepsilon}\right\|_{1, \varepsilon}^{2} \leq C(1+t) M \varepsilon^{2} C_{\varepsilon}^{2} / 2
$$

and the result follows.

Lemma 6.4. Keeping the notations of the proof of Theorem 6.3, the following formula holds $\overline{\mathbb{P}}_{x}^{\varepsilon}$ almost surely:

$$
d \varphi_{\varepsilon}\left(\bar{X}_{t}^{\varepsilon}, X_{t}^{\varepsilon}\right)=\varepsilon^{-2} \boldsymbol{L}^{\varepsilon} \varphi_{\varepsilon}\left(\bar{X}_{t}^{\varepsilon}, X_{t}^{\varepsilon}\right) d t+\varepsilon^{-1} \sigma^{*}\left(D+\varepsilon \partial_{y}\right) \varphi_{\varepsilon}\left(\bar{X}_{t}^{\varepsilon}, X_{t}^{\varepsilon}\right) d B_{t} .
$$

Proof: To simplify the notations, choose $\varepsilon=1$ and omit the parameter $\varepsilon$ (as a consequence $\varphi_{\varepsilon}$ is denoted by $\varphi$ ). Once again, we follow [6, Sect 8.3]. The method is quite similar to Section 5 so that we just outline the main ideas.

As in Section $5, e_{k}(1 \leq k \leq d)$ still stands for the $\mathrm{k}$-th vector of the canonical basis of $\mathbb{R}^{d}$. For any $\boldsymbol{\psi} \in L^{2}\left(\Omega \times \mathbb{R}^{\bar{d}} ; \Pi\right)$ and $r \in \mathbb{R}^{*}$, we define $\left[\Gamma_{r}^{k} \boldsymbol{\psi}\right](\omega, y)=\frac{1}{r}\left[\boldsymbol{\psi}\left(\tau_{r e_{k}} \omega, y+r e_{k}\right)-\right.$ $\boldsymbol{\psi}(\omega, y)]$. We have again: $\left(\Gamma_{r}^{k} \boldsymbol{\phi}, \boldsymbol{\psi}\right)_{2, \Pi}=-\left(\boldsymbol{\phi}, \Gamma_{-r}^{k} \boldsymbol{\psi}\right)_{2, \Pi}$, for any $\boldsymbol{\phi}, \boldsymbol{\psi} \in L^{2}\left(\Omega \times \mathbb{R}^{d} ; \Pi\right)$, and $\left|\Gamma_{r}^{k} \phi\right|_{2, \Pi} \leq\left|\left(D+\partial_{y}\right) \phi\right|_{2, \Pi}$ for any $\boldsymbol{\phi} \in \mathbb{H}_{\Pi}$.

Following the proof of Proposition 5.1, we can prove that $\left(D+\partial_{y}\right) \varphi \in \mathbb{H}_{\Pi}$ so that it makes sense to consider $\left(D+\partial_{y}\right)^{2} \boldsymbol{\varphi} \in L^{2}\left(\Omega \times \mathbb{R}^{d} ; \Pi\right)$.

Let us now consider a mollifier $\varrho: \mathbb{R}^{d} \rightarrow \mathbb{R}$ with compact support and define, for $n \geq 1$, $\varrho_{n}=n^{d} \varrho(n \cdot)$. Then we define $\varphi^{n}(\omega, y)=\int_{\mathbb{R}^{d}} \varphi\left(\tau_{-x^{\prime}} \omega, y-x^{\prime}\right) \varrho_{n}\left(x^{\prime}\right) d x^{\prime}$. Again with classical convolution technics we can establish that $\left(\varphi^{n}\right)_{n}$ (resp. $\left(\left(D+\partial_{y}\right) \varphi^{n}\right)_{n}$, resp. $((D+$ $\left.\left.\partial_{y}\right)^{2} \boldsymbol{\varphi}^{n}\right)_{n}$ ) converges to $\boldsymbol{\varphi}$ (resp. $\left(D+\partial_{y}\right) \boldsymbol{\varphi}$, resp. $\left.\left(D+\partial_{y}\right)^{2} \boldsymbol{\varphi}\right)$ in $L^{2}\left(\Omega \times \mathbb{R}^{d} ; \Pi\right)$ as $n$ goes to $\infty$. Moreover the function $x \in \mathbb{R}^{d} \mapsto \varphi^{n}\left(\tau_{x} \omega, x\right)$ is of class $C^{2}$. Thus we can apply the Itô formula to this function and to the process $X$, and then pass to the limit as $n$ goes to $\infty$ with the help of Lemma 5.2 and an adapted version of Lemma 5.3, as explained in Section 5. The result then follows.

\section{Homogenization Property}

This section is devoted to the presentation and the proof of the homogenization property. We remind the reader that $\boldsymbol{u}_{\lambda}^{i}$ stands for the solution of the auxiliary problems, that the solution 
of the resolvent equation $\lambda \boldsymbol{u}_{\lambda}^{i}(\cdot, y)-\boldsymbol{L}^{y} \boldsymbol{u}_{\lambda}^{i}(\cdot, y)=\boldsymbol{b}_{i}(\cdot, y)$ (see Section 4). Moreover, for $i=1, \ldots, d$ and $y \in \mathbb{R}^{d}$ fixed, the functions $\left(D \boldsymbol{u}_{\lambda}^{i}(\cdot, y)\right)_{\lambda}$ are convergent in $L^{2}(\Omega)^{d}$ towards $\boldsymbol{\xi}_{i}(\cdot, y)$ and are bounded in $L^{2}(\Omega)$-norm independently of $y$ and $\lambda$. It thus makes sense to define, for every $y \in \mathbb{R}^{d}$,

$$
\begin{aligned}
& \bar{A}(y)=\lim _{\lambda \rightarrow 0} \mathbb{M}\left[\left(I+D \boldsymbol{u}_{\lambda}\right)^{*} \boldsymbol{a}\left(I+D \boldsymbol{u}_{\lambda}\right)(., y)\right]=\mathbb{M}\left[(I+\boldsymbol{\xi})^{*} \boldsymbol{a}(I+\boldsymbol{\xi})(., y)\right], \\
& \bar{H}(y)=\lim _{\lambda \rightarrow 0} \mathbb{M}\left[\left(I+D \boldsymbol{u}_{\lambda}\right)^{*} \boldsymbol{H}\left(I+D \boldsymbol{u}_{\lambda}\right)(., y)\right]=\mathbb{M}\left[(I+\boldsymbol{\xi})^{*} \boldsymbol{H}(I+\boldsymbol{\xi})(., y)\right], \\
& \bar{B}(y)=(1 / 2) \partial_{y}[\bar{A}+\bar{H}](y) .
\end{aligned}
$$

Thanks to Proposition 4.3, all these coefficients are twice continuously differentiable with bounded derivatives up to order two. In particular, it makes sense to consider the strong solution of the following SDE

$$
X_{t}=x+\int_{0}^{t} \bar{B}\left(X_{r}\right) d r+\int_{0}^{t} \bar{A}^{1 / 2}\left(X_{r}\right) d B_{r} .
$$

As observed in [14], one of the consequences of (32) is the tightness of the process $X^{\varepsilon}$ in $C\left([0, T] ; \mathbb{R}^{d}\right)$. We will establish that each converging subsequence of $\left(X^{\varepsilon}\right)_{\varepsilon}$ solves the martingale problem (41). As a consequence, Theorem 3.1 holds.

Proof of Theorem 3.1: Let us consider a converging subsequence of $\left(X^{\varepsilon}\right)_{\varepsilon}$, still indexed with $\varepsilon$. We just have to identify the finite dimensional distributions of the limit.

Applying Theorem 5.4 to the function $\boldsymbol{u}_{\lambda}$ and choosing $\lambda=\varepsilon^{2}$ yields

$$
\begin{aligned}
d X_{t}^{\varepsilon}= & -\varepsilon d u_{\varepsilon^{2}}\left(\bar{X}_{t}^{\varepsilon}, X_{t}^{\varepsilon}\right)+\varepsilon\left(\partial_{y} u_{\varepsilon^{2}}\right)^{*} \sigma\left(\bar{X}_{t}^{\varepsilon}, X_{t}^{\varepsilon}\right) d B_{t} \\
& +\left[\varepsilon u_{\varepsilon^{2}}+\varepsilon c \cdot \partial_{y} u_{\varepsilon^{2}}+(\varepsilon / 2) \operatorname{trace}\left(a \partial_{y y}^{2} u_{\varepsilon^{2}}\right)\right]\left(\bar{X}_{t}^{\varepsilon}, X_{t}^{\varepsilon}\right) d t \\
& +\left[b \partial_{y} u_{\varepsilon^{2}}+c \cdot\left(I+D u_{\varepsilon^{2}}\right)+\operatorname{trace}\left(a D \partial_{y} u_{\varepsilon^{2}}\right)\right]\left(\bar{X}_{t}^{\varepsilon}, X_{t}^{\varepsilon}\right) d t \\
& +\left[\sigma+D u_{\varepsilon^{2}}^{*} \sigma\right]\left(\bar{X}_{t}^{\varepsilon}, X_{t}^{\varepsilon}\right) d B_{t} \\
\equiv & d \Theta_{t}^{1, \varepsilon}+d \Theta_{t}^{2, \varepsilon}+d \Theta_{t}^{3, \varepsilon}+d \Theta_{t}^{4, \varepsilon}
\end{aligned}
$$

From Proposition 4.3 and Lemma 5.2, it is plain to see that $\overline{\mathbb{E}}_{x}^{\varepsilon}\left[\left(\Theta_{t}^{1, \varepsilon}\right)^{2}+\left(\Theta_{t}^{2, \varepsilon}\right)^{2}\right] \rightarrow 0$ as $\varepsilon$ goes to 0 . Let us now focus now on $\Theta_{t}^{4, \varepsilon}$. The quadratic variations exactly match $\int_{0}^{t}[\mathrm{I}+$ $\left.D u_{\varepsilon^{2}}\right]^{*} a\left[\mathrm{I}+D u_{\varepsilon^{2}}\right]\left(\bar{X}_{r}^{\varepsilon}, X_{r}^{\varepsilon}\right) d r$ and satisfy $\overline{\mathbb{E}}_{x}^{\varepsilon}\left[\sup _{0 \leq s \leq t}\left|<\Theta^{4, \varepsilon}>_{s}-\int_{0}^{s} \bar{A}\left(X_{r}^{\varepsilon}\right) d r\right|\right] \rightarrow 0$ as $\varepsilon$ goes to 0 (Theorem 6.1). Combining this with Proposition 7.1 below, the limit is readily identified as the solution of SDE (41). So we complete the proof of Theorem 3.1.

Proposition 7.1. The following convergence holds: $\overline{\mathbb{E}}_{x}^{\varepsilon}\left[\left|\Theta_{t}^{3, \varepsilon}-\int_{0}^{t} \bar{B}\left(X_{r}^{\varepsilon}\right) d r\right|^{2}\right] \rightarrow 0$ as $\varepsilon$ tends to 0. 
Proof: The main difficulty actually lies in the term corresponding to $b \partial_{y} u_{\varepsilon^{2}}$. The other terms are easily treated with the help of Theorem 6.1 and the convergence of the derivatives $\left(D \boldsymbol{u}_{\lambda}\right)_{\lambda}$ and $\left(D \partial_{y} \boldsymbol{u}_{\lambda}\right)_{\lambda}$ stated in Proposition 4.3. The strategy consists in establishing that, up to introducing new correctors for $\boldsymbol{b} \partial_{y} \boldsymbol{u}_{\varepsilon^{2}}$, this term can be divided in two parts, the former satisfying Theorem 6.1 and the latter satisfying Theorem 6.3.

We keep the notations of the proof of Theorem 6.3 and use the convention of summation over repeated indices. To find these correctors, let us consider a test function $\varphi \in \mathcal{C}_{\Pi}$. Proceeding with successive integration by parts, we have, for $1 \leq i \leq d$,

$$
\begin{aligned}
2\left(\boldsymbol{b} \partial_{y} \boldsymbol{u}_{\varepsilon^{2}}^{i}, \boldsymbol{\varphi}\right)_{2, \Pi}= & \left(D_{j}(\boldsymbol{a}+\boldsymbol{H})_{j k} \partial_{y_{k}} \boldsymbol{u}_{\varepsilon^{2}}^{i}, \boldsymbol{\varphi}\right)_{2, \Pi} \\
= & -\left((\boldsymbol{a}+\boldsymbol{H})_{j k} \partial_{y_{k}} \boldsymbol{u}_{\varepsilon^{2}}^{i}, D_{j} \boldsymbol{\varphi}\right)_{2, \Pi}-\left((\boldsymbol{a}+\boldsymbol{H})_{j k} D_{j} \partial_{y_{k}} \boldsymbol{u}_{\varepsilon^{2}}^{i}, \boldsymbol{\varphi}\right)_{2, \Pi} \\
= & -\left((\boldsymbol{a}+\boldsymbol{H})_{j k} \partial_{y_{k}} \boldsymbol{u}_{\varepsilon^{2}}^{i},\left(D_{j}+\varepsilon \partial_{y_{j}}\right) \boldsymbol{\varphi}\right)_{2, \Pi}-\left((\boldsymbol{a}+\boldsymbol{H})_{j k} D_{j} \partial_{y_{k}} \boldsymbol{u}_{\varepsilon^{2}}^{i}, \boldsymbol{\varphi}\right)_{2, \Pi} \\
& +\left((\boldsymbol{a}+\boldsymbol{H})_{j k} \partial_{y_{k}} \boldsymbol{u}_{\varepsilon^{2}}^{i}, \varepsilon \partial_{y_{j}} \boldsymbol{\varphi}\right)_{2, \Pi} \\
= & -\left((\boldsymbol{a}+\boldsymbol{H})_{j k} \partial_{y_{k}} \boldsymbol{u}_{\varepsilon^{2}}^{i},\left(D_{j}+\varepsilon \partial_{y_{j}}\right) \boldsymbol{\varphi}\right)_{2, \Pi}-\left((\boldsymbol{a}+\boldsymbol{H})_{j k} D_{j} \partial_{y_{k}} \boldsymbol{u}_{\varepsilon^{2}}^{i}, \boldsymbol{\varphi}\right)_{2, \Pi} \\
& -\varepsilon\left(\partial_{y_{j}}(\boldsymbol{a}+\boldsymbol{H})_{j k} \partial_{y_{k}} \boldsymbol{u}_{\varepsilon^{2}}^{i}, \boldsymbol{\varphi}\right)_{2, \Pi}-\varepsilon\left((\boldsymbol{a}+\boldsymbol{H})_{j k} \partial_{y_{k} y_{j}}^{2} \boldsymbol{u}_{\varepsilon^{2}}^{i}, \boldsymbol{\varphi}\right)_{2, \Pi}
\end{aligned}
$$

The decomposition and the correctors now appear clearly. Define the correctors as

$$
\operatorname{Corr}^{i, \varepsilon}=(\varepsilon / 2) \partial_{y_{j}}(\boldsymbol{a}+\boldsymbol{H})_{j k} \partial_{y_{k}} \boldsymbol{u}_{\varepsilon^{2}}^{i}+(\varepsilon / 2)(\boldsymbol{a}+\boldsymbol{H})_{j k} \partial_{y_{k} y_{j}}^{2} \boldsymbol{u}_{\varepsilon^{2}}^{i},
$$

the converging part in $L^{2}\left(\Omega \times \mathbb{R}^{d} ; \widetilde{\mathbb{M}}_{5.2}^{t}\right)$

$$
\operatorname{Conv}^{i, \varepsilon}=-(1 / 2)(\boldsymbol{a}+\boldsymbol{H})_{j k} D_{j} \partial_{y_{k}} \boldsymbol{u}_{\varepsilon^{2}}^{i}
$$

and the exploding part that we aim to treat with Theorem 6.3

$$
\operatorname{Expl}^{i, \varepsilon}=\boldsymbol{b} \partial_{y} \boldsymbol{u}_{\varepsilon^{2}}^{i}+\operatorname{Corr}^{i, \varepsilon}-\operatorname{Conv}^{i, \varepsilon} .
$$

Clearly, from Assumptions 2.2 and 2.3 and Proposition 4.3, the family $\left(\mathrm{Corr}^{i, \varepsilon}\right)_{\varepsilon}$ converges to 0 in $L^{2}\left(\Omega \times \mathbb{R}^{d} ; \widetilde{\mathbb{M}}_{5.2}^{t}\right)$ to 0 . Then Lemma 5.2 ensures that

$$
\overline{\mathbb{E}}_{x}^{\varepsilon}\left[\left|\int_{0}^{t} \operatorname{Corr}^{i, \varepsilon}\left(\bar{X}_{r}^{\varepsilon}, X_{r}^{\varepsilon}\right) d r\right|\right] \rightarrow 0, \quad \text { as } \varepsilon \rightarrow 0 .
$$

From Proposition 4.3 and Theorem 6.1, we can prove that $\overline{\mathbb{E}}_{x}^{\varepsilon}\left[\mid \int_{0}^{t} \operatorname{Conv}^{i, \varepsilon}\left(\bar{X}_{r}^{\varepsilon}, X_{r}^{\varepsilon}\right) d r-\right.$ $\left.\int_{0}^{t} \bar{K}\left(X_{r}^{\varepsilon}\right) d r \mid\right] \rightarrow 0$ as $\varepsilon$ tends to 0, where $\bar{K}(y)=\mathbb{M}\left[-(1 / 2)(\boldsymbol{a}+\boldsymbol{H})_{j k} \partial_{y_{k}} \boldsymbol{\xi}_{i}(\cdot, y)\right]$.

It remains to treat the part $\mathrm{Expl}^{i, \varepsilon}$, for which we have already proved that

$$
\left(\operatorname{Expl}^{i, \varepsilon}, \boldsymbol{\varphi}\right)_{2, \Pi}=-(1 / 2)\left((\boldsymbol{a}+\boldsymbol{H})_{j k} \partial_{y_{k}} \boldsymbol{u}_{\varepsilon^{2}}^{i},\left(D_{j}+\varepsilon \partial_{y_{j}}\right) \boldsymbol{\varphi}\right)_{2, \Pi} .
$$


We aim at applying Theorem 6.3. The only technical obstacle is that the function $\operatorname{Expl}^{i, \varepsilon}$ need not satisfy $\int_{\mathbb{R}^{d}}\left|\operatorname{Expl}^{i, \varepsilon}(\cdot, y)\right|_{2}^{2} d y<+\infty$. However, it can be easily overcome. Indeed, it seems natural to introduce a nonnegative smooth function $\varrho: \mathbb{R}^{d} \rightarrow \mathbb{R}$ with compact support and to define

$$
\operatorname{Expl}_{\varrho}^{i, \varepsilon}(\omega, y)=\operatorname{Expl}^{i, \varepsilon}(, y) \varrho(y)+(\varepsilon / 2)(\boldsymbol{a}+\boldsymbol{H})_{j k} \partial_{y_{k}} \boldsymbol{u}_{\varepsilon^{2}}^{i} \partial_{y_{j}} \varrho .
$$

Obviously, $\int_{\mathbb{R}^{d}}\left|\operatorname{Expl}_{\rho}^{i, \varepsilon}(\cdot, y)\right|_{2}^{2} d y<+\infty$. Furthermore, from Lemma 5.2 and Assumption 2.2,

$$
\begin{aligned}
\overline{\mathbb{E}}_{x}^{\varepsilon}\left[\left|\int_{0}^{t}\left[\operatorname{Expl}^{i, \varepsilon}-\operatorname{Expl}_{\rho}^{i, \varepsilon}\right]\left(\bar{X}_{r}^{\varepsilon}, X_{r}^{\varepsilon}\right) d r\right|\right] \leq & 2 \Lambda \sup _{y \in \mathbb{R}^{d}}\left|\partial_{y} \varrho(y)\right| \widetilde{\mathbb{M}}_{5.2}^{t}\left[\left|\varepsilon \partial_{y_{k}} \boldsymbol{u}_{\varepsilon^{2}}^{i}\right|\right] \\
& +\sup _{y \in \mathbb{R}^{d}}\left|\operatorname{Expl}^{i, \varepsilon}(\cdot, y)\right|_{2} \widetilde{\mathbb{M}}_{5.2}^{t}[|1-\varrho(y)|] .
\end{aligned}
$$

From (42) and (26a), $\sup _{y \in \mathbb{R}^{d}}\left|\operatorname{Expl}^{i, \varepsilon}(\cdot, y)\right|_{2}$ is finite and bounded by a constant that depends neither on $\varepsilon$ nor on $y \in \mathbb{R}^{d}$ and the family $\left(\widetilde{\mathbb{M}}_{5.2}^{t}\left[\left|\varepsilon \partial_{y_{k}} \boldsymbol{u}_{\varepsilon^{2}}^{i}\right|\right]\right)_{\varepsilon}$ converges to 0 as $\varepsilon$ tends to 0 . Moreover, from (43) and (44), for a test function $\varphi \in \mathcal{C}_{\Pi}$, it is readily seen that

$$
\begin{aligned}
\left(\operatorname{Expl}_{\rho}^{i, \varepsilon}, \boldsymbol{\varphi}\right)_{2, \Pi}= & \left(\operatorname{Expl}^{i, \varepsilon}, \boldsymbol{\varphi} \varrho\right)_{2, \Pi}+(\varepsilon / 2)\left((\boldsymbol{a}+\boldsymbol{H})_{j k} \partial_{y_{k}} \boldsymbol{u}_{\varepsilon^{2}}^{i} \partial_{y_{j}} \varrho, \boldsymbol{\varphi}\right)_{2, \Pi} \\
= & -(1 / 2)\left((\boldsymbol{a}+\boldsymbol{H})_{j k} \partial_{y_{k}} \boldsymbol{u}_{\varepsilon^{2}}^{i},\left(D_{j}+\varepsilon \partial_{y_{j}}\right)(\boldsymbol{\varphi} \varrho)\right)_{2, \Pi} \\
& +(\varepsilon / 2)\left((\boldsymbol{a}+\boldsymbol{H})_{j k} \partial_{y_{k}} \boldsymbol{u}_{\varepsilon^{2}}^{i} \partial_{y_{j}} \varrho, \boldsymbol{\varphi}\right)_{2, \Pi} \\
= & -(1 / 2)\left(\varrho(\boldsymbol{a}+\boldsymbol{H})_{j k} \partial_{y_{k}} \boldsymbol{u}_{\varepsilon^{2}}^{i},\left(D_{j}+\varepsilon \partial_{y_{j}}\right) \boldsymbol{\varphi}\right)_{2, \Pi} \\
\leq & C_{\varepsilon}\left(\mathbb{M} \int_{\mathbb{R}^{d}}\left|\left(D+\varepsilon \partial_{y}\right) \boldsymbol{\varphi}(., y)\right|^{2} d y\right)^{1 / 2}
\end{aligned}
$$

where $C_{\varepsilon}=(1 / 2)(M+\Lambda) \int_{\mathbb{R}^{d}}\left|\varrho(y) \partial_{y} \boldsymbol{u}_{\varepsilon^{2}}^{i}(\cdot, y)\right|_{2} d y$. From Proposition 4.3, $\varepsilon C_{\varepsilon} \rightarrow 0$ as $\varepsilon \rightarrow 0$ so that Theorem 6.3 can now be applied. Consequently, $\overline{\mathbb{E}}_{x}^{\varepsilon}\left[\left|\int_{0}^{t} \operatorname{Expl}_{\rho}^{i, \varepsilon}\left(\bar{X}_{r}^{\varepsilon}, X_{r}^{\varepsilon}\right) d r\right|\right] \rightarrow 0$ as $\varepsilon$ tends to 0 , for each fixed function $\varrho$. Hence, we just have to fix a function $\varrho$ in order for the second term in the right-hand side of (45) to be small and then to choose $\varepsilon$ small enough to make the first term and $\int_{0}^{t} \operatorname{Expl}_{\rho}^{i, \varepsilon}\left(\bar{X}_{r}^{\varepsilon}, X_{r}^{\varepsilon}\right) d r$ small. The result follows.

It just remain to check that the limit can be expressed in terms of $\bar{B}$, as stated in Proposition 7.1. All we know at present is $\overline{\mathbb{E}}_{x}^{\varepsilon}\left[\left|\Theta_{t}^{3, \varepsilon}-\int_{0}^{t} \bar{F}\left(X_{r}^{\varepsilon}\right) d r\right|^{2}\right] \rightarrow 0$ as $\varepsilon$ tends to 0 , where the entries $\bar{F}_{i}, 1 \leq i \leq d$, of $\bar{F}$ are given by ( $\delta$ stands for the Kroenecker symbol)

$$
\left.\bar{F}_{i}=\mathbb{M}\left[\boldsymbol{c}_{j}\left(\delta_{i j}+\boldsymbol{\xi}_{i j}\right)+\boldsymbol{a}_{j k} \partial_{y_{k}} \boldsymbol{\xi}_{i j}-(1 / 2)(\boldsymbol{a}+\boldsymbol{H})_{j k} \partial_{y_{k}} \boldsymbol{\xi}_{i j}\right)(\cdot, y)\right] .
$$


Hence

$$
\begin{aligned}
\bar{F}_{i} & \left.=\mathbb{M}\left[(1 / 2) \partial_{y_{k}}(\boldsymbol{a}+\boldsymbol{H})_{k j}\left(\delta_{i j}+\boldsymbol{\xi}_{i j}\right)+\boldsymbol{a}_{k j} \partial_{y_{k}} \boldsymbol{\xi}_{i j}-(1 / 2)(\boldsymbol{a}-\boldsymbol{H})_{k j} \partial_{y_{k}} \boldsymbol{\xi}_{i j}\right)(\cdot, y)\right] \\
& \left.=\mathbb{M}\left[(1 / 2) \partial_{y_{k}}(\boldsymbol{a}+\boldsymbol{H})_{k j}\left(\delta_{i j}+\boldsymbol{\xi}_{i j}\right)+(1 / 2)(\boldsymbol{a}+\boldsymbol{H})_{k j} \partial_{y_{k}} \boldsymbol{\xi}_{i j}\right)(\cdot, y)\right] \\
& =(1 / 2) \partial_{y_{k}} \mathbb{M}\left[(\boldsymbol{a}+\boldsymbol{H})_{k j}\left(\delta_{i j}+\boldsymbol{\xi}_{i j}\right)(\cdot, y)\right] .
\end{aligned}
$$

The proof is over if we can prove that $(\bar{A}+\bar{H})(y)=\mathbb{M}[(\boldsymbol{a}+\boldsymbol{H})(I+\boldsymbol{\xi})(\cdot, y)]$. Choosing $\boldsymbol{\varphi}=\boldsymbol{u}_{\lambda}^{j}(\cdot, y)$ in (25) and passing to the limit as $\lambda$ goes to 0 yields $\left((\boldsymbol{a}+\boldsymbol{H})\left(e_{i}+\boldsymbol{\xi}_{i}\right), \boldsymbol{\xi}_{j}\right)_{2}=0$, that is $\mathbb{M}\left[\boldsymbol{\xi}^{*}(\boldsymbol{a}+\boldsymbol{H})(I+\boldsymbol{\xi})(\cdot, y)\right]=0$. We now complete the proof.

Acknowledgments. The author is thankful to Stefano Olla for fruitful discussions about this topic and to an anonymous referee for a careful reading of the paper.

\section{References}

[1] A. Benchérif-Madani, É. Pardoux, Homogenization of a diffusion with locally periodic coefficients, in Séminaire de Probabilités, Lecture Notes in Mathematics, to appear.

[2] A. Bensoussan, J.L. Lions , G. Papanicolaou, Asymptotic methods in periodic media, Ed. North Holland, 1978.

[3] F. Delarue, R. Rhodes, Stochastic Homogenization of Quasilinear PDEs with a Spatial Degeneracy, submitted, available at http://hal.archives-ouvertes.fr/hal-00141167.

[4] A. De Masi, P. Ferrari, S. Goldstein, W.D. Wick, An invariance principle for reversible Markov processes. Application to random motions in random environments, J. Stat. Phys., 1989, 55, p. 787-855.

[5] M. Fukushima, Y. Oshima , M. Takeda, Dirichlet Forms and Symmetric Markov Processes, De Gruyter Studies in Mathematics 19, Walter de Gruyter, Berlin and Hawthorne, New York, 1994.

[6] D. Gilbarg, N.S. Trudinger, Elliptic partial differential equations of second order, Grundlehren der mathematischen Wissenschaft, 1977, 224, Springer.

[7] V.V. Jikov, S.M. Kozlov, O.A. Oleinik, Homogenization of differential operators and integral functionals, Springer-Verlag, Berlin, 1994.

[8] C. Kipnis, S.R.S. Varadhan, Central limit theorem for additive functionals of reversible Markov processes and application to simple exclusion, Ann. Probab.,2000, 28 , no. 1 , 277302. 
[9] S.M. Kozlov, The method of averaging and walks in inhomogeneous environments, Russian Math. Surveys, 1985, 40, 2, p. 73-145.

[10] Z.M. Ma, M. Röckner, Introduction to the Theory of (Non-Symmetric) Dirichlet Forms, Universitext, Berlin Heidelberg, Springer-Verlag, 1992.

[11] S. Olla, Homogenization of diffusion processes in Random Fields, Cours de l'école doctorale, Ecole polytechnique, 1994.

[12] S. Olla, P. Siri, Homogenization of a bond diffusion in a locally ergodic random environment, Stochastic Processes and Applications, 2004, vol 109, 317-326.

[13] H. Osada, Diffusion processes with generators of generalized divergence form, J. Math. Kyoto Univ., 1987, 27, 597-619.

[14] G. Papanicolaou, S.R.S. Varadhan, Boundary Value Problems with rapidly oscillating random coefficients, Coll. Math. Soc. János Bolyai, 1979, 27, Random Fields, p. 835-873. 